To be published in International Journal of Damage Mechanics

as a special issue of ESMC2009 (Lisbon, 2009

Article submitted on 25 november 2009, accepted on 16 february 2010 (23 pages)

\title{
THE EFFECTIVE BEHAVIOR OF ELASTIC BODIES CONTAINING MICROCRACKS OR MICROHOLES LOCALIZED ON A SURFACE
}

\author{
JEAN-JACQUES MARIGO* AND CATHERINE PIDERI
}

\begin{abstract}
We propose a two-scale method to find the effective behavior of a three-dimensional linear elastic medium containing a series of microcracks or microholes located on a surface. The obtained effective behavior is that of a homogeneous body with, in place of the actual microdefects, a surface across which the displacements and the stresses suffer jump discontinuities. The transmission conditions are in general of Ventcel's type. The coefficients entering in these jump conditions are obtained by solving six elastic problems posed on an infinite representative cell. The theoretical analysis is illustrated by a few examples.
\end{abstract}

\section{INTRODUCTION AND NOTATIONS}

This paper is devoted to the study of the influence of localized (by opposition to diffuse) defects on the overall response of linearly elastic bodies. Typical examples of such "damage" distributions can be seen in Figure 1: on the left figure, we see a family of micro-holes localized on a (plane) surface of a homogeneous body; in the middle, the localized defects are transverse micro-cracks in the matrix of a fiber-reinforced composite; on the right, the defects are an array of holes whose size is larger than that of the population of holes existing in the bulk. Since the
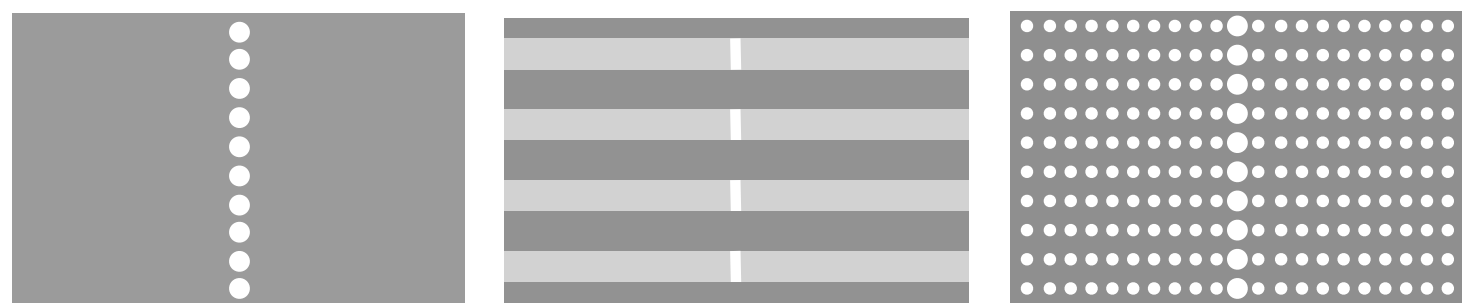

FiguRE 1. Some examples of defects localized on a surface

defects are not distributed in all or a large part of the body, we cannot use the classical methods and results of the homogenization theory, cf Sanchez-Palencia (1980), Léné (1984) or Suquet (1987). The influence of the defects cannot be represented by an effective rigidity tensor as it

Key words and phrases. two-scale method, asymptotic analysis, matched asymptotic expansions, homogenization, microcracks, microholes, damage, Ventcel's conditions.

* corresponding author. 
is the case when the defects are periodically distributed in the body (or by an effective rigidity tensor field depending on the local density of defects when the distribution is only "almost" periodic) like in Marigo (1985), Andrieux et al. (1986) or Devries et al. (1989). Intuitively, that gives rise to boundary layer effects because of the local loss of the homogeneity or of the periodicity of the heterogeneity of the material. It should be quite similar to what happens at the boundary of a periodic medium, cf Dumontet (1990).

That means that our problem is closer to that of finding the influence of a thin heterogeneous layer inside a homogeneous body. There exists a large number of papers devoted to this type of problems when the layer itself is assumed to be elastic and homogeneous. In most part they use asymptotic methods based on the presence of at least one small parameter (the thinness of the layer). That leads to a large variety of asymptotic models depending on the assumptions made on the behavior of the layer and the possible presence of another small parameters, cf Abdelmoula et al. (1998), Benveniste (2006), Benveniste and Miloh (2001), Bessoud et al. (2009), Bessoud et al. (2008), Caillerie (1980), Geymonat et al. (1999), Huy and SanchezPalencia (1974), Klarbring and Movchan (1998), Krasucki and Lenci (2000b), Krasucki and Lenci (2000a), Licht and Michaille (1997). The "limit" model essentially consists in finding effective transmission conditions across the "interface" (the surface where the layer is located). However, in order that non trivial transmission conditions appear at the first order (i.e. when the displacements or the stress vector are discontinuous), it is necessary to assume that the layer is either soft or stiff in comparison to the material in the bulk. In other words, it is necessary that the ratio between the stiffness of the layer and the stiffness of the material in the bulk could be considered as either a small or a large parameter. In such cases the transmissions conditions can be divided into two types: (i) Robin's conditions, (ii) Ventcel's conditions. In the former case, the stress vector is continuous at the interface and the jump of the displacements is proportional (in linear elasticity) to the stress vector. This corresponds to the case when the layer is soft. In the latter case, considered by Ventcel (1959), both the stress vector and the displacements are discontinuous at the interface and the jump of the stress tensor depends on the tangential derivatives of the mean values of the stress components at the interface, cf Huy and Sanchez-Palencia (1974), Caillerie (1980), Lemrabet (1987). This corresponds to the case when the layer is stiff.

In our case of localized defects, if we assume that they are periodically distributed on a surface, the unique small parameter is the characteristic length of the period. The immediate consequence is that the defects have only second order effects (typical boundary layer effects). Therefore, one needs to make an asymptotic analysis at least up to the second order. Moreover, due to the fact that the heterogeneity is no more "layered", it is necessary to develop an asymptotic method coupling the local character of the heterogeneity with its periodical character. The ideal candidate is the matched asymptotic expansion technique, first introduced in a similar context by Nguetseng (1985), Nguetseng and Sanchez-Palencia (1986) and Sanchez-Palencia (1986). Except for these pioneering works, there exist very few papers devoted to these types of localized defects, see Abdelmoula and Marigo (2000) or Bilteryst and Marigo (2003). The present paper is in the development of these approaches. Among those described in Figure 1, we will consider the simplest case, but in a full three-dimensional anisotropic setting. Accordingly, 
we will consider the cases of micro-holes or micro-cracks periodically distributed on a surface of a homogeneous linear elastic body. The goal is to obtain the local effective behavior of the body, that is the transmission conditions induced by the micro-defects, and to study the influence of the shape of the defects, of the period and of the anisotropy of the material.

The paper is organized as follows. In Section 2 we set the problem in which the body contains a family of micro-defects periodically distributed on a cross-section, the characteristic period length being the small parameter. Then, in Section 3, we make an asymptotic analysis of this problem based on a matched asymptotic expansion method. Specifically, we assume that the displacement and stress fields admit two asymptotic expansions, the outer one far from the surface where the defects are located and the inner one near that surface. Then we construct the outer and inner problems giving the different terms of the expansions. We show that the order 0 outer problem is that of a sound body, the defects being "invisible" at this order. The defects become visible in the order 1 outer problem, their presence leading to Ventcel-type transmission conditions across the surface where the defects are located. Those Ventcel's transmission conditions involve two effective tensors whose coefficients are given by six elementary inner problems posed on a representative cell of the localized defects. The section 4 is devoted to the study of those transmission conditions and on the effective tensors entering in their definition. We show that the two effective tensors can be deduced from a symmetric fourth order tensor which can be interpreted as the effective damage compliance tensor of the representative cell with holes. These general results (established in a full anisotropic context) are illustrated by three examples.

The summation convention on repeated indices is implicitly adopted in the sequel. The vectors and second order tensors are indicated by boldface letters, like $\mathbf{u}$ and $\boldsymbol{\sigma}$ for the displacement field and the stress field. Their components are denoted by italic letters, like $u_{i}$ and $\sigma_{i j}$. The third or fourth order tensors as well as their components are indicated by a sans serif letter, like $\mathrm{A}$ or $A_{i j k l}$ for the stiffness tensor. Such tensors are considered as linear maps applying on vectors or second order tensors and the application is denoted without dots, like $A \varepsilon$ whose $i j$-component is $\mathrm{A}_{i j k l} \varepsilon_{k l}$. The inner product between two vectors or two tensors of the same order is indicated by a dot, like $\mathbf{a} \cdot \mathbf{b}$ which stands for $a_{i} b_{i}$ or $\boldsymbol{\sigma} \cdot \boldsymbol{\varepsilon}$ for $\sigma_{i j} \varepsilon_{i j}$. The symbol $\otimes$ denotes the tensor product and $\otimes_{s}$ its symmetrized, i.e. $2 \mathbf{e}_{1} \otimes_{s} \mathbf{e}_{2}=\mathbf{e}_{1} \otimes \mathbf{e}_{2}+\mathbf{e}_{2} \otimes \mathbf{e}_{1}$.

As we make ample use of multiple scaling techniques, we adopt related notation. For instance, $\mathbf{x}=\left(x_{1}, x_{2}, x_{3}\right)$ always denotes a macroscopic coordinate while $\mathbf{y}=\left(y_{1}, y_{2}, y_{3}\right)$ will represent a microscopic one. Since the defects are located near the plane $x_{1}=0$, we distinguish the normal coordinates $x_{1}$ and $y_{1}$ from the tangential coordinates $\mathbf{x}^{\prime}=\left(x_{2}, x_{3}\right)$ and $\mathbf{y}^{\prime}=\left(y_{2}, y_{3}\right)$. The Latin indices run from 1 to 3 , while the Greek indices run from 2 to 3 . When a spatial (scalar, vectorial or tensorial) field depends both on $\mathbf{x}$ and $\mathbf{y}$, the partial derivative with respect to one of these coordinates appears explicitly as an index : for example, $\boldsymbol{d i v}_{\mathbf{x}} \boldsymbol{\tau}$ and $\varepsilon_{\mathbf{x}}(\mathbf{v})$ denote respectively the divergence of the stress tensor field $\boldsymbol{\tau}$ and the symmetrized gradient of the vector field $\mathbf{v}$ 
with respect to $\mathbf{x}$, while $\operatorname{div}_{\mathbf{y}} \boldsymbol{\tau}$ and $\varepsilon_{\mathbf{y}}(\mathbf{v})$ are the corresponding derivatives with respect to $\mathbf{y}$ :

$$
\begin{gathered}
\operatorname{div}_{\mathbf{x}} \boldsymbol{\tau}(\mathbf{x}, \mathbf{y}) \equiv \frac{\partial \tau_{i j}}{\partial x_{j}}(\mathbf{x}, \mathbf{y}) \mathbf{e}_{i} \quad, \quad \operatorname{div}_{\mathbf{y}} \boldsymbol{\tau}(\mathbf{x}, \mathbf{y}) \equiv \frac{\partial \tau_{i j}}{\partial y_{j}}(\mathbf{x}, \mathbf{y}) \mathbf{e}_{i} \\
2 \varepsilon_{\mathbf{x}}(\mathbf{v})_{i j}(\mathbf{x}, \mathbf{y}) \equiv \frac{\partial v_{j}}{\partial x_{i}}(\mathbf{x}, \mathbf{y})+\frac{\partial v_{i}}{\partial x_{j}}(\mathbf{x}, \mathbf{y}) \quad, \quad 2 \varepsilon_{\mathbf{y}}(\mathbf{v})_{i j}(\mathbf{x}, \mathbf{y}) \equiv \frac{\partial v_{i}}{\partial y_{j}}(\mathbf{x}, \mathbf{y})+\frac{\partial v_{j}}{\partial y_{i}}(\mathbf{x}, \mathbf{y})
\end{gathered}
$$

On a surface $\boldsymbol{\Gamma}$ across which a field $f$ is discontinuous, we will denote by $f^{+}$and $f^{-}$its right and left limits and by $\llbracket f \rrbracket$ its jump discontinuity. For instance, when $\boldsymbol{\Gamma}$ is a subset of the plane $x_{1}=0$, we set :

$$
f^{ \pm}\left(\mathbf{x}^{\prime}\right)=f\left(0 \pm, \mathbf{x}^{\prime}\right) \equiv \lim _{h \downarrow 0} f\left( \pm h, \mathbf{x}^{\prime}\right), \quad \llbracket f \rrbracket\left(\mathbf{x}^{\prime}\right) \equiv f^{+}\left(\mathbf{x}^{\prime}\right)-f^{-}\left(\mathbf{x}^{\prime}\right) .
$$

\section{The aCtual Elastic Problem on the Damaged Body}

We consider a three-dimensional elastic body with natural reference configuration the bounded regular connected open subset $\boldsymbol{\Omega}$ of $\mathbb{R}^{3}$ and boundary $\partial \boldsymbol{\Omega}$. We denote by $\mathbf{e}_{1}, \mathbf{e}_{2}, \mathbf{e}_{3}$ the canonical basis of $\mathbb{R}^{3}$ and by $\left(x_{1}, x_{2}, x_{3}\right)$ the associated cartesian coordinates. The body is occupied by a linear elastic material, not necessarily isotropic, characterized by its symmetric positive stiffness tensor A. The cross-section of the body in the plane $x_{1}=0$ is denoted by $\boldsymbol{\Gamma}$. The body is weakened by a set of identical micro-holes or micro-cracks whose centers are periodically distributed on $\boldsymbol{\Gamma}$, cf Figure 2. The two vectors defining the periodicity are $\epsilon \mathbf{a}$ and $\epsilon \mathbf{b}$ where $\mathbf{a}$ and $\mathbf{b}$ are two vectors of the plane $\mathbf{e}_{2}$ and $\mathbf{e}_{3}$, and $\epsilon$ is a small length (in comparison with the diameter $L$ of $\boldsymbol{\Gamma}$ ) characterizing the size of the defects. In the sequel $\epsilon$ will be considered as a small parameter. At the micro-scale $\mathbf{y}=\mathbf{x} / \epsilon$, the representative cell of the body in the neighborhood of $\boldsymbol{\Gamma}$ is a parallelepiped $\mathbf{Y}$ infinite in the direction $y_{1}$, with a parallelogram $\mathbf{P}$ defined by the vectors $\mathbf{a}$ and $\mathbf{b}$ as the cross-section $($ i.e. $\mathbf{Y}=\mathbb{R} \times \mathbf{P}$ ) and containing a hole or a crack $\mathcal{D}$ (compact subset of $\mathbf{Y}$ ), cf Figure 3. Note that $\mathcal{D}$ is not necessarily connected, that means that $\mathcal{D}$ can be the union of a family of compact connected components. In other words, $\mathcal{D}$ can correspond to several holes or cracks. With an abuse of language, we will always qualify $\mathcal{D}$ as the hole or the crack of the cell. The parameter $\epsilon$ is chosen so that the area of $\mathbf{P}$ be equal to 1 . The sound part $\mathbf{Y} \backslash \mathcal{D}$ of the cell is supposed connected (in other words, the holes or the cracks do not separate the body into two parts). The union of all the micro-defects is denoted by $\mathcal{D}^{\epsilon}$ and accordingly the sound part of the body is $\Omega \backslash \mathcal{D}^{\epsilon}$. In the sequel, we will establish the fundamental results in the case where the defect is a hole, that is the case where $0<\operatorname{vol}(\mathcal{D})<+\infty$. (More precisely, we will assume that $\mathcal{D}$ is closed and bounded in $\mathbf{Y}$, is the closure of its interior and $\operatorname{vol}(\mathcal{D})>0$.) The case of a crack, when $\operatorname{vol}(\mathcal{D})=0$, could be treated in a same manner with minor changes. We will simply indicate at the end of the construction what differs in such a case. The body is submitted to prescribed displacements on the part $\partial_{D} \boldsymbol{\Omega}$ of $\partial \boldsymbol{\Omega}$, to prescribed surface forces on the complementary part $\partial_{F} \boldsymbol{\Omega}$, while the body forces are assumed to be zero. Moreover the surfaces of the micro-holes are free. Since the holes can intersect the boundary $\partial \boldsymbol{\Omega}$, we will assume that the surface forces $\mathbf{F}$ are prescribed to 0 in a neighborhood of $\partial \boldsymbol{\Gamma}$. 


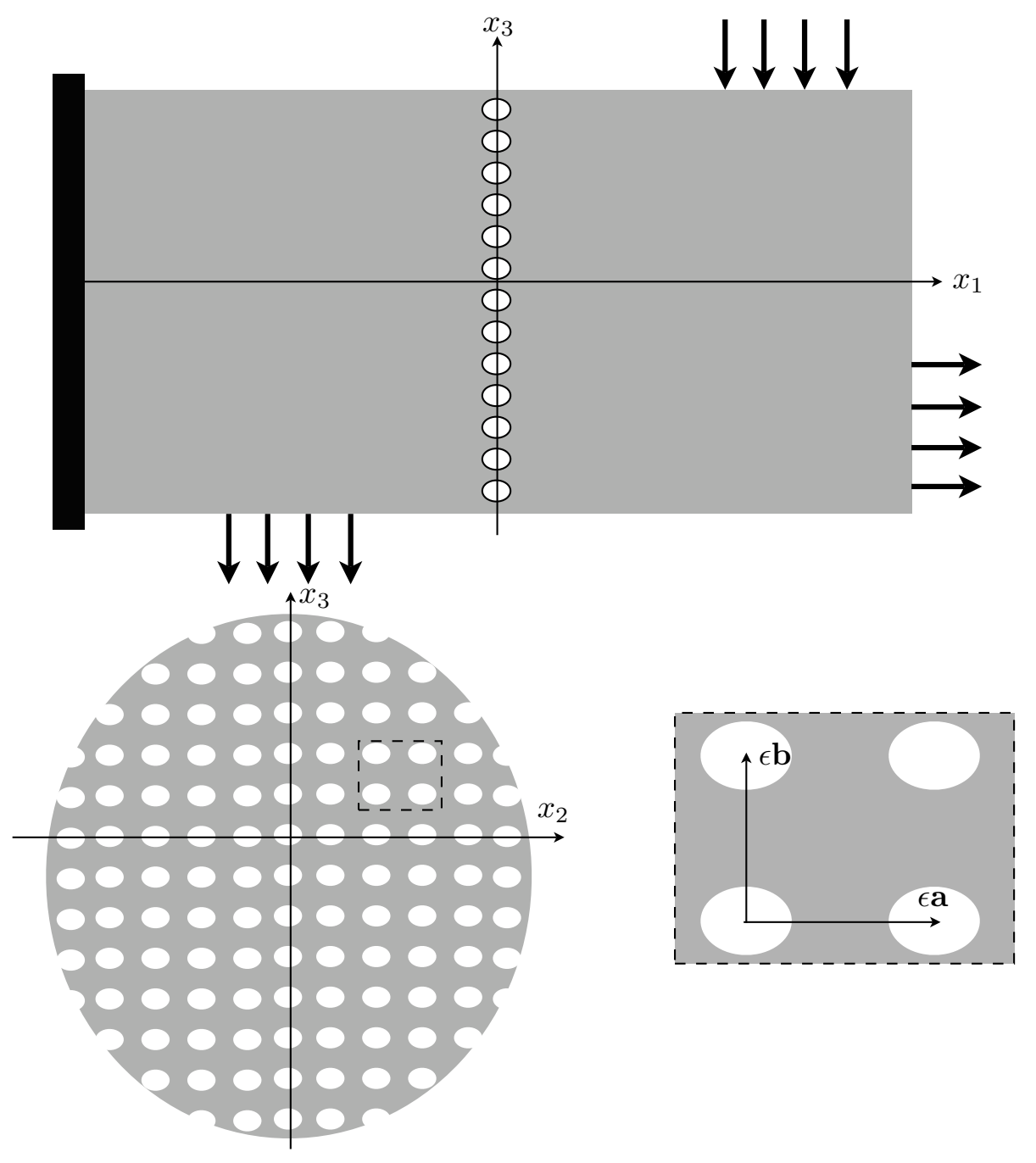

Figure 2. The body with the localized set of micro-defects. Top: the section $x_{2}=0$; Bottom : the cross-section $\boldsymbol{\Gamma}\left(x_{1}=0\right)$ where the micro-holes are centered.

Accordingly, at equilibrium, the displacement field and the stress field resulting in the damaged body are $\left(\mathbf{u}^{\epsilon}, \boldsymbol{\sigma}^{\epsilon}\right)$ - where the dependence with respect to the parameter $\epsilon$ appears explicitly - and have to satisfy the following set of equations, referred to in the sequel as the real 


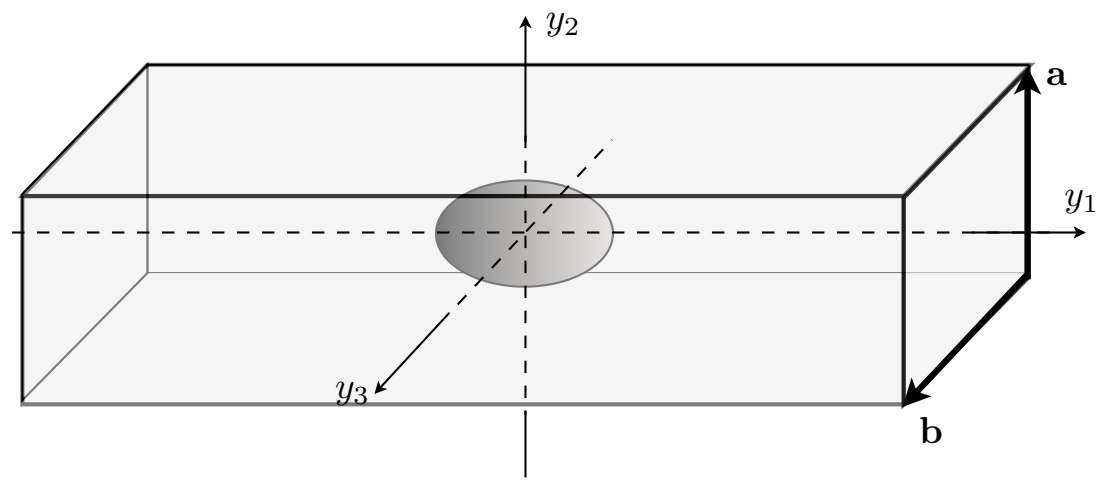

Figure 3. The infinite representative cell $\mathbf{Y}=\mathbb{R} \times \mathbf{P}$ with one hole $\mathcal{D}$.

problem:

$$
\begin{array}{ll}
\text { Equilibrium in } \boldsymbol{\Omega} \backslash \mathcal{D}^{\epsilon} & : \operatorname{div} \boldsymbol{\sigma}^{\epsilon}=\mathbf{0}, \\
\text { Constitutive relation in } \boldsymbol{\Omega} \backslash \mathcal{D}^{\epsilon} & : \boldsymbol{\sigma}^{\epsilon}=\mathrm{A} \boldsymbol{\varepsilon}\left(\mathbf{u}^{\epsilon}\right), \\
\text { Boundary conditions on } \partial \mathcal{D}^{\epsilon} & : \boldsymbol{\sigma}^{\epsilon} \mathbf{n}=\mathbf{0}, \\
\text { Boundary conditions on } \partial_{F} \boldsymbol{\Omega} & : \boldsymbol{\sigma}^{\epsilon} \mathbf{n}=\mathbf{F}, \\
\text { Boundary conditions on } \partial_{D} \boldsymbol{\Omega} & : \mathbf{u}^{\epsilon}=\mathbf{u}^{\mathbf{d}} .
\end{array}
$$

The first equation of (4) corresponds to the equilibrium, the second one to the anisotropic Hooke law, the third one expresses that the holes are free of forces and the two last ones are the boundary conditions, $\mathbf{n}$ denoting generically the unit outer normal. Using classical results of the linear elasticity theory, the real problem (4) admits a unique solution.

\section{Asymptotic ANALYSis}

Since the number of defects is large or equivalently the parameter $\epsilon$ is small, we propose to deduce an approximation of the real fields $\left(\mathbf{u}^{\epsilon}, \boldsymbol{\sigma}^{\epsilon}\right)$ from their asymptotic behavior when $\epsilon$ goes to 0 .

3.1. The forms of the asymptotic expansions. Following a classical process, see Nguetseng and Sanchez-Palencia (1986) or Sanchez-Palencia (1986), we assume that $\left(\mathbf{u}^{\epsilon}, \boldsymbol{\sigma}^{\epsilon}\right)$ can be expanded by using two asymptotic expansions, the first one - called the outer asymptotic expansion - valid far from $\boldsymbol{\Gamma}$, site of the micro-holes, and the second - called the inner asymptotic expansion - valid in the neighborhood of $\boldsymbol{\Gamma}$. Moreover, assuming that both expansions hold true in an intermediary zone, these two expansions are matched by the so-called matching conditions. 
(1) Outer expansion. Far from $\boldsymbol{\Gamma}$, that is when $\left|x_{1}\right| \gg \epsilon$, we assume that $\left(\mathbf{u}^{\epsilon}, \boldsymbol{\sigma}^{\epsilon}\right)$ can be expanded as

$$
\mathbf{u}^{\epsilon}(\mathbf{x})=\sum_{i=0}^{\infty} \epsilon^{i} \mathbf{u}^{i}(\mathbf{x}), \quad \boldsymbol{\sigma}^{\epsilon}(\mathbf{x})=\sum_{i=0}^{\infty} \epsilon^{i} \boldsymbol{\sigma}^{i}(\mathbf{x}) .
$$

(2) Inner expansion. Near $\boldsymbol{\Gamma}$, that is when $\left|x_{1}\right| \ll L$, we assume that $\left(\mathbf{u}^{\epsilon}, \boldsymbol{\sigma}^{\epsilon}\right)$ can be expanded as

$$
\mathbf{u}^{\epsilon}(\mathbf{x})=\sum_{i=0}^{\infty} \epsilon^{i} \mathbf{v}^{i}\left(\mathbf{x}^{\prime}, \frac{\mathbf{x}}{\epsilon}\right), \quad \boldsymbol{\sigma}^{\epsilon}(\mathbf{x})=\sum_{i=0}^{\infty} \epsilon^{i} \boldsymbol{\tau}^{i}\left(\mathbf{x}^{\prime}, \frac{\mathbf{x}}{\epsilon}\right),
$$

where the $\mathbf{v}^{i}$ 's and the $\boldsymbol{\tau}^{i}$ 's are functions of $\left(\mathbf{x}^{\prime}, y_{1}, \mathbf{y}^{\prime}\right)$, periodic, with period $\mathbf{a}$ and $\mathbf{b}$, with respect to the transversal microscopic variable $\mathbf{y}^{\prime}$, i.e. :

$$
\begin{array}{ll}
\mathbf{v}^{i}\left(\mathbf{x}^{\prime}, y_{1}, \mathbf{y}^{\prime}+p \mathbf{a}+q \mathbf{b}\right)=\mathbf{v}^{i}\left(\mathbf{x}^{\prime}, y_{1}, \mathbf{y}^{\prime}\right) & \forall p, q \in \mathbb{Z}^{2} . \\
\boldsymbol{\tau}^{i}\left(\mathbf{x}^{\prime}, y_{1}, \mathbf{y}^{\prime}+p \mathbf{a}+q \mathbf{b}\right)=\boldsymbol{\tau}^{i}\left(\mathbf{x}^{\prime}, y_{1}, \mathbf{y}^{\prime}\right) & \forall p, q \in \mathbb{Z}^{2} .
\end{array}
$$

(3) Matching conditions. Since the inner and the outer expansions (5)-(6) are both valid when $\epsilon \ll\left|x_{1}\right| \ll L$, they have to satisfy the following matching conditions : for $i \in \mathbb{N}$,

$$
\begin{aligned}
& \lim _{y_{1} \rightarrow \pm \infty}\left(\mathbf{v}^{i}\left(\mathbf{x}^{\prime}, \mathbf{y}\right)-\sum_{j=0}^{i} \frac{y_{1}^{i-j}}{(i-j) !} \frac{\partial^{i-j} \mathbf{u}^{j}}{\partial x_{1}^{i-j}}\left(0 \pm, \mathbf{x}^{\prime}\right)\right)=\mathbf{0} \\
& \lim _{y_{1} \rightarrow \pm \infty}\left(\boldsymbol{\tau}^{i}\left(\mathbf{x}^{\prime}, \mathbf{y}\right)-\sum_{j=0}^{i} \frac{y_{1}^{i-j}}{(i-j) !} \frac{\partial^{i-j} \boldsymbol{\sigma}^{j}}{\partial x_{1}^{i-j}}\left(0 \pm, \mathbf{x}^{\prime}\right)\right)=\mathbf{0}
\end{aligned}
$$

with the convention that $\partial^{0} f / \partial x^{0}=f$ and $f\left(0 \pm, \mathbf{x}^{\prime}\right)$ stands for $f^{ \pm}\left(\mathbf{x}^{\prime}\right)$, cf $(3)$. In the sequel, we will only use the first two $(i=0,1)$ which can read as

$$
\begin{gathered}
\lim _{y_{1} \rightarrow \pm \infty} \mathbf{v}^{0}\left(\mathbf{x}^{\prime}, \mathbf{y}\right)=\mathbf{u}^{0 \pm}\left(\mathbf{x}^{\prime}\right), \\
\lim _{y_{1} \rightarrow \pm \infty} \boldsymbol{\tau}^{0}\left(\mathbf{x}^{\prime}, \mathbf{y}\right)=\boldsymbol{\sigma}^{0 \pm}\left(\mathbf{x}^{\prime}\right), \\
\lim _{y_{1} \rightarrow \pm \infty}\left(\mathbf{v}^{1}\left(\mathbf{x}^{\prime}, \mathbf{y}\right)-\mathbf{u}^{1 \pm}\left(\mathbf{x}^{\prime}\right)-y_{1} \frac{\partial \mathbf{u}^{0}}{\partial x_{1}}\left(0 \pm, \mathbf{x}^{\prime}\right)\right)=0, \\
\lim _{y_{1} \rightarrow \pm \infty}\left(\boldsymbol{\tau}^{1}\left(\mathbf{x}^{\prime}, \mathbf{y}\right)-\boldsymbol{\sigma}^{1 \pm}\left(\mathbf{x}^{\prime}\right)-y_{1} \frac{\partial \boldsymbol{\sigma}^{0}}{\partial x_{1}}\left(0 \pm, \mathbf{x}^{\prime}\right)\right)=0 .
\end{gathered}
$$

Remark 1. The outer terms $\mathbf{u}^{i}$ and $\boldsymbol{\sigma}^{i}$ have to be defined on the whole body $\boldsymbol{\Omega}$ even if the expansions (5) hold true only far away from $\boldsymbol{\Gamma}$. The transmission conditions across $\boldsymbol{\Gamma}$ are not known in advance. We have to deduce them from the matching conditions. It is one of the key points of the study.

The inner terms $\mathbf{v}^{i}$ and $\boldsymbol{\tau}^{i}$ have only to be defined on $\mathbf{\Gamma} \times \mathbf{Y}$. Indeed, owing to (7)-(8), they are extended by periodicity to $\boldsymbol{\Gamma} \times \mathbb{R}^{3}$. However, $\mathbf{v}^{i}$ and $\boldsymbol{\tau}^{i}$ are not defined a priori in the holed parts $\boldsymbol{\Gamma} \times \mathcal{D}$. It can be useful to extend them inside the holes so that they be defined in the whole domain $\mathbf{\Gamma} \times \mathbf{Y}$. We will explain how later. The behavior of $\mathbf{v}^{i}$ and $\boldsymbol{\tau}^{i}$ at infinity in the direction 1 are not known in advance. That behavior will be also deduced from the matching conditions. 
As we shall see in the subsequent analysis, neither the outer expansion (5), nor the inner one (6) are valid at (and near) the boundary curve $\partial \mathbf{\Gamma}$. A third form of the expansions should be used, but, in order to simplify the exposition and because the required information does not call for such an expansion, we do not concern ourselves with it.

The main goal of the paper is to determine $\mathbf{u}^{0}, \boldsymbol{\sigma}^{0}, \mathbf{u}^{1}$ and $\boldsymbol{\sigma}^{1}$. That requires to determine also $\mathbf{v}^{0}, \mathbf{v}^{1}$ and $\boldsymbol{\tau}^{0}$.

3.2. The family of equations governing the outer and inner terms. We insert the assumed expansions of $\mathbf{u}^{\epsilon}$ and $\boldsymbol{\sigma}^{\epsilon}$ in the set of equations of the real problem and obtain :

$$
\begin{array}{cl}
\operatorname{div} \boldsymbol{\sigma}^{\epsilon}(\mathbf{x})=\sum_{i=0}^{\infty} \epsilon^{i} \operatorname{div} \boldsymbol{\sigma}^{i}(\mathbf{x}) \quad, \quad \operatorname{div} \boldsymbol{\sigma}^{\epsilon}(\mathbf{x})=\sum_{i=0}^{\infty} \epsilon^{i}\left(\operatorname{div}_{\mathbf{x}} \tau^{i}\left(\mathbf{x}^{\prime}, \mathbf{y}\right)+\frac{1}{\epsilon} \operatorname{div}_{\mathbf{y}} \tau^{i}\left(\mathbf{x}^{\prime}, \mathbf{y}\right)\right), \\
\varepsilon\left(\mathbf{u}^{\epsilon}\right)(\mathbf{x})=\sum_{i=0}^{\infty} \epsilon^{i} \varepsilon\left(\mathbf{u}^{i}\right)(\mathbf{x}) \quad, \quad \varepsilon\left(\mathbf{u}^{\epsilon}\right)(\mathbf{x})=\sum_{i=0}^{\infty} \epsilon^{i}\left(\varepsilon_{\mathbf{x}}\left(\mathbf{v}^{i}\right)\left(\mathbf{x}^{\prime}, \mathbf{y}\right)+\frac{1}{\epsilon} \varepsilon_{\mathbf{y}}\left(\mathbf{v}^{i}\right)\left(\mathbf{x}^{\prime}, \mathbf{y}\right)\right)
\end{array}
$$

Identifying the terms with the same power of $\epsilon$ yields a set of equations which govern the inner and outer terms, namely,

\section{Equilibrium equations:}

\begin{tabular}{l|l|l} 
Order & Outer : in $\boldsymbol{\Omega} \backslash \boldsymbol{\Gamma}$ & Inner : in $\boldsymbol{\Gamma} \times(\mathbf{Y} \backslash \mathcal{D})$ \\
\hline-1 & none & $\mathbf{0}=\operatorname{div}_{\mathbf{y}} \boldsymbol{\tau}^{0}$ \\
$i \geq 0$ & $\mathbf{0}=\operatorname{div} \boldsymbol{\sigma}^{i}$ & $\mathbf{0}=\operatorname{div}_{\mathbf{y}} \boldsymbol{\tau}^{i+1}+\boldsymbol{d i v}_{\mathbf{x}} \boldsymbol{\tau}^{i}$
\end{tabular}

\section{Constitutive relations:}

\begin{tabular}{l|l|l} 
Order & Outer : in $\boldsymbol{\Omega} \backslash \boldsymbol{\Gamma}$ & Inner : in $\boldsymbol{\Gamma} \times(\mathbf{Y} \backslash \mathcal{D})$ \\
\hline-1 & none & $\mathbf{0}=\mathrm{A} \varepsilon_{\mathbf{y}}\left(\mathbf{v}^{0}\right)$ \\
$i \geq 0$ & $\boldsymbol{\sigma}^{i}=\mathrm{A} \boldsymbol{\varepsilon}\left(\mathbf{u}^{i}\right)$ & $\boldsymbol{\tau}^{i}=\mathrm{A} \varepsilon_{\mathbf{y}}\left(\mathbf{v}^{i+1}\right)+\mathrm{A} \boldsymbol{\varepsilon}_{\mathbf{x}}\left(\mathbf{v}^{i}\right)$
\end{tabular}

\begin{tabular}{|c|c|c|}
\hline Order & Outer & Inner \\
\hline 0 & 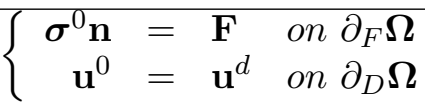 & $\boldsymbol{\tau}^{0} \mathbf{n}=\mathbf{0}$ on $\boldsymbol{\Gamma} \times \partial \mathcal{D}$ \\
\hline$i \geq 1$ & 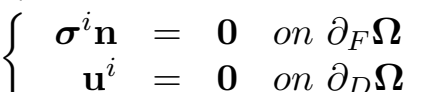 & $\boldsymbol{\tau}^{i} \mathbf{n}=\mathbf{0}$ on $\boldsymbol{\Gamma} \times \partial \mathcal{D}$ \\
\hline
\end{tabular}

\section{Boundary conditions:}

Of course, we must add to this set of equations, at any order, the periodic conditions (7)-(8) satisfied by $\mathbf{v}^{i}$ and $\boldsymbol{\tau}^{i}$.

Remark 2. As we said in Remark 1, it is useful to extend the definition of $\boldsymbol{\tau}^{i}$ to the whole cell $\mathbf{Y}$. The natural way is to extend $\boldsymbol{\tau}^{i}$ by $\mathbf{0}$ inside $\mathcal{D}$. Accordingly, the inner equilibrium equations can read as

$$
\operatorname{div}_{\mathbf{y}} \tau^{i+1}+\operatorname{div}_{\mathbf{x}} \tau^{i}=\mathbf{0} \quad \text { in } \quad \boldsymbol{\Gamma} \times \mathbf{Y}
$$


and the boundary conditions on the surface of the hole are automatically verified. In the sequel, when $\boldsymbol{\tau}^{i}$ needs to be extended, we implicitly assume that we have chosen that extension.

Addition of these relations to the matching conditions (9) and (10) yields a set of equations coupling inner and outer terms. Through an inductive process, the next subsections are devoted to a partial solving of this set of equations so as to get the first two terms of the inner and outer expansions for displacements and stresses, namely $\mathbf{u}^{0}, \mathbf{u}^{1}, \mathbf{v}^{0}, \mathbf{v}^{1}, \boldsymbol{\sigma}^{0}, \boldsymbol{\sigma}^{1}$ and $\boldsymbol{\tau}^{0}$.

3.3. Determination of $\mathbf{u}^{0}, \boldsymbol{\sigma}^{0}$ and $\mathbf{v}^{0}$. From the constitutive relations of order -1 , we get $\varepsilon_{\mathbf{y}}\left(\mathbf{v}^{0}\right)=\mathbf{0}$ and, by virtue of the periodic conditions, $\mathbf{v}^{0}$ does not depend on $\mathbf{y}$. Then, the matching condition (11) yields both the continuity of $\mathbf{u}^{0}$ across $\boldsymbol{\Gamma}$ and the relation between $\mathbf{v}^{0}$ and $\mathbf{u}^{0}$, namely,

$$
\begin{gathered}
\llbracket \mathbf{u}^{0} \rrbracket=\mathbf{0} \quad \text { on } \boldsymbol{\Gamma}, \\
\mathbf{v}^{0}\left(\mathbf{x}^{\prime}, \mathbf{y}\right)=\mathbf{u}^{0}\left(0, \mathbf{x}^{\prime}\right) .
\end{gathered}
$$

Let us prove now the continuity of the stress vector $\boldsymbol{\sigma}^{0} \mathbf{e}_{1}$ across $\boldsymbol{\Gamma}$, i.e.

$$
\llbracket \boldsymbol{\sigma}^{0} \rrbracket \mathbf{e}_{1}=\mathbf{0} \quad \text { on } \boldsymbol{\Gamma}
$$

First integrate over the holed cell $\mathbf{Y} \backslash \mathcal{D}$ the inner equilibrium equation of order -1 , then use both the periodic conditions and the boundary condition on the boundary of the hole to obtain

$$
\int_{\mathbf{P}}\left(\boldsymbol{\tau}^{0}\left(\mathbf{x}^{\prime},+\infty, \mathbf{y}^{\prime}\right) \mathbf{e}_{1}-\boldsymbol{\tau}^{0}\left(\mathbf{x}^{\prime},-\infty, \mathbf{y}^{\prime}\right) \mathbf{e}_{1}\right) d \mathbf{y}^{\prime}=\mathbf{0} .
$$

On the other hand, integrating over the cell cross-section $\mathbf{P}$ and recalling that $\operatorname{area}(\mathbf{P})=1$, the matching condition (12) yields

$$
\int_{\mathbf{P}} \boldsymbol{\tau}^{0}\left(\mathbf{x}^{\prime}, \pm \infty, \mathbf{y}^{\prime}\right) \mathbf{e}_{1} d \mathbf{y}^{\prime}=\boldsymbol{\sigma}^{0 \pm}\left(\mathbf{x}^{\prime}\right) \mathbf{e}_{1},
$$

and the desired result follows by difference of the two limits. Therefore we have

Proposition 1. $\left(\mathbf{u}^{0}, \boldsymbol{\sigma}^{0}\right)$ is the unique solution of the elastic problem set on a sound body:

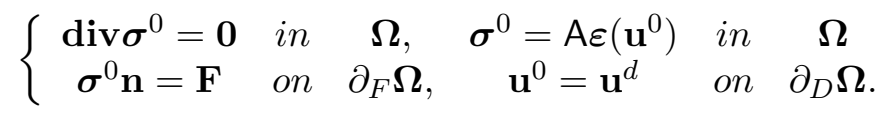

Remark 3. In other words, the holes are not visible at the first order, they have only second order effects. This result is classical but fundamental, cf Nguetseng (1985), Nguetseng and Sanchez-Palencia (1986) or Abdelmoula and Marigo (2000), it could be entitled "the principle of the dressmaker", that is to say, "it is not necessary to sew entirely to pieces of fabrics in order to render invisible their relative opening, it is sufficient to sew them at a great number of points regularly spaced". It is a major difference with the case usually considered in the literature of a thin layer made of either a soft or a stiff material (by comparison to the material in the bulk). In such cases, the effects of the layer are visible at the first order and lead to either Robin's type 
transmission conditions in the soft case or Ventcel's type transmission conditions in the stiff case, see Geymonat et al. (1999), Bessoud et al. (2008).

At this stage, $\mathbf{u}^{0}, \mathbf{v}^{0}$ and $\boldsymbol{\sigma}^{0}$ are (in principle) determined.

3.4. Determination of $\mathbf{v}^{1}$ and $\tau^{0}$. The inner terms $\mathbf{v}^{1}$ and $\tau^{0}$ have to satisfy the inner equilibrium of order $-1\left(\mathbf{d i v}_{\mathbf{y}} \boldsymbol{\tau}^{0}=\mathbf{0}\right)$, the inner constitutive relation of order $0\left(\boldsymbol{\tau}^{0}=A \varepsilon_{\mathbf{x}}\left(\mathbf{v}^{0}\right)+\right.$ $\left.\mathrm{A} \varepsilon_{y}\left(\mathbf{v}^{1}\right)\right)$, the vanishing stress condition on the boundary of the hole $\left(\boldsymbol{\tau}^{0} \mathbf{n}=\mathbf{0}\right)$, the periodic conditions on the faces of the cell and the conditions at $y_{1}= \pm \infty$ furnished by the matching conditions of order 0 for the stresses $\left(\boldsymbol{\tau}^{0}\left(\mathbf{x}^{\prime}, \pm \infty, \mathbf{y}^{\prime}\right)=\boldsymbol{\sigma}^{0}\left(0, \mathbf{x}^{\prime}\right)\right)$. This leads to a $\mathbf{x}^{\prime}$-indexed family of elastic problems on the holed cell $\mathbf{Y} \backslash \mathcal{D}$. They can be rewritten by introducing as new unknowns the following fields $\hat{\mathbf{v}}$ and $\hat{\boldsymbol{\tau}}$,

$$
\begin{aligned}
\mathbf{v}^{1}\left(\mathbf{x}^{\prime}, \mathbf{y}\right) & =y_{1} \frac{\partial \mathbf{u}^{0}}{\partial x_{1}}\left(0, \mathbf{x}^{\prime}\right)+\hat{\mathbf{v}}\left(\mathbf{x}^{\prime}, \mathbf{y}\right) \\
\boldsymbol{\tau}^{0}\left(\mathbf{x}^{\prime}, \mathbf{y}\right) & =\boldsymbol{\sigma}^{0}\left(0, \mathbf{x}^{\prime}\right)+\hat{\boldsymbol{\tau}}\left(\mathbf{x}^{\prime}, \mathbf{y}\right)
\end{aligned}
$$

which are interpreted as the boundary layer correctors for the displacements and the stresses near the cross-section $\boldsymbol{\Gamma}$ due to the presence of the microholes. By virtue of (19) and after remarking that

$$
\varepsilon_{\mathbf{y}}\left(y_{1} \frac{\partial \mathbf{u}^{0}}{\partial x_{1}}\left(0, \mathbf{x}^{\prime}\right)\right)+\varepsilon_{\mathbf{x}}\left(\mathbf{v}^{0}\right)\left(\mathbf{x}^{\prime}\right)=\varepsilon\left(\mathbf{u}^{0}\right)\left(0, \mathbf{x}^{\prime}\right)
$$

a straightforward calculation shows that $\hat{\mathbf{v}}$ and $\hat{\boldsymbol{\tau}}$ have to satisfy

$$
\left\{\begin{array}{lll}
\operatorname{div}_{\mathbf{y}} \hat{\boldsymbol{\tau}}=\mathbf{0} & \text { in } & \boldsymbol{\Gamma} \times(\mathbf{Y} \backslash \mathcal{D}) \\
\hat{\boldsymbol{\tau}}=\mathrm{A} \varepsilon_{\mathbf{y}}(\hat{\mathbf{v}}) & \text { in } & \boldsymbol{\Gamma} \times(\mathbf{Y} \backslash \mathcal{D}) \\
\hat{\mathbf{v}} \text { and } \hat{\boldsymbol{\tau}} \mathbf{n} \text { periodic } & \text { on } & \boldsymbol{\Gamma} \times \partial \mathbf{Y} \\
\hat{\boldsymbol{\tau}}=\mathbf{0} & \text { on } & \boldsymbol{\Gamma} \times\{ \pm \infty\} \times \mathbf{P} \\
\hat{\boldsymbol{\tau}}\left(\mathbf{x}^{\prime}, \mathbf{y}\right) \mathbf{n}(\mathbf{y})=-\boldsymbol{\sigma}^{0}\left(0, \mathbf{x}^{\prime}\right) \mathbf{n}(\mathbf{y}) & \text { on } & \boldsymbol{\Gamma} \times \partial \mathcal{D}
\end{array}\right.
$$

Let us note that this problem is akin to finding the response of the holed cell when the surface of the hole is submitted to surface forces. This type of linear elastic problem on an infinite strip has been studied time and time again, and its solution is by now well known. Therefore, we merely recall its main features and refer the interested reader to the specialized literature for the details of the proofs, see for example Sanchez-Palencia (1986) or Dumontet (1990) and references therein.

(1) For a given value of $\mathbf{x}^{\prime} \in \boldsymbol{\Gamma},(25)$ admits a solution $(\hat{\mathbf{v}}, \hat{\boldsymbol{\tau}})$, unique for $\hat{\boldsymbol{\tau}}$ and unique up to a ( $\mathbf{x}^{\prime}$-dependent) translation for $\hat{\mathbf{v}}$;

(2) The stress field $\hat{\boldsymbol{\tau}}$ exponentially decays to zero when $\left|y_{1}\right|$ goes to infinity, while the displacement field $\hat{\mathbf{v}}$ exponentially tends to a ( $\mathbf{x}^{\prime}$-dependent) translation when $y_{1}$ goes to $+\infty$, and to another $\left(\mathbf{x}^{\prime}\right.$-dependent) translation when $y_{1}$ goes to $-\infty$. 
By linearity, (25) can be divided into six "elementary" problems (elementary in the sense that they can be solved once and for all for a given cell $\mathbf{Y}$ and a given hole $\mathcal{D}$, because they are independent of $\mathbf{x}^{\prime}$ and of the value of $\boldsymbol{\sigma}^{0}$ on $\boldsymbol{\Gamma}$ ). Specifically, we obtain the following

Proposition 2. $\hat{\mathbf{v}}$ and $\hat{\boldsymbol{\tau}}$ can be decomposed into :

$$
\begin{aligned}
& \hat{\mathbf{v}}\left(\mathbf{x}^{\prime}, \mathbf{y}\right)=\sigma_{i j}^{0}\left(0, \mathbf{x}^{\prime}\right) \mathbf{V}^{i j}(\mathbf{y})+\check{\mathbf{v}}\left(\mathbf{x}^{\prime}\right), \\
& \hat{\boldsymbol{\tau}}\left(\mathbf{x}^{\prime}, \mathbf{y}\right)=\sigma_{i j}^{0}\left(0, \mathbf{x}^{\prime}\right) \mathbf{T}^{i j}(\mathbf{y}),
\end{aligned}
$$

where $\check{\mathbf{v}}$ stands for the (still arbitrary) $\mathbf{x}^{\prime}$-dependent vector field, while $\left(\mathbf{V}^{i j}, \mathbf{T}^{i j}\right)=\left(\mathbf{V}^{j i}, \mathbf{T}^{j i}\right)$ denote the solutions to the elementary inner problems,

$\left\{\begin{array}{lll}\operatorname{div} \mathbf{T}^{i j}=\mathbf{0} & \text { in } & \mathbf{Y} \backslash \mathcal{D} \\ \mathbf{T}^{i j}=\mathrm{A} \varepsilon\left(\mathbf{V}^{i j}\right) & \text { in } & \mathbf{Y} \backslash \mathcal{D} \\ \mathbf{V}^{i j} \text { and } \mathbf{T}^{i j} \mathbf{n} \text { periodic } & \text { on } & \mathbb{R} \times \partial \mathbf{P} \\ \mathbf{T}^{i j}=\mathbf{0} & \text { on } & \{ \pm \infty\} \times \mathbf{P} \\ \mathbf{T}^{i j} \mathbf{n}=-\frac{1}{2}\left(n_{j} \mathbf{e}_{i}+n_{i} \mathbf{e}_{j}\right) & \text { on } & \partial \mathcal{D}\end{array}\right.$.

Remark 4. In the spirit of Remark 2, we can extend the definition of $\mathbf{T}^{i j}$ to the whole cell $\mathbf{Y}=\mathbb{R} \times \mathbf{P}$ by setting

$$
2 T_{k l}^{i j}=-\left(\delta_{i k} \delta_{j l}+\delta_{i l} \delta_{j k}\right) \quad \text { in } \mathcal{D}
$$

where $\boldsymbol{\delta}$ denotes the Kronecker symbol. Accordingly, we have $\mathbf{d i v} \mathbf{T}^{i j}=\mathbf{0}$ in $\mathbf{Y}$ and the boundary condition on $\partial \mathcal{D}$ is automatically satisfied.

These six problems enjoy the same properties as (25): $\mathbf{T}^{i j}$ exponentially decays to zero and $\mathbf{V}^{i j}$ tends to a constant vector, say $\mathbf{V}^{i j \pm}$, when $y_{1}$ goes to $\pm \infty$,

$$
\mathbf{V}^{i j+} \equiv \lim _{y_{1} \rightarrow+\infty} \mathbf{V}^{i j}(\mathbf{y}), \quad \mathbf{V}^{i j-} \equiv \lim _{y_{1} \rightarrow-\infty} \mathbf{V}^{i}(\mathbf{y})
$$

Since $\mathbf{V}^{i j}$ is unique up to a constant vector, the latter is determined by introducing the additional condition

$$
\mathbf{V}^{i j+}+\mathbf{V}^{i j-}=\mathbf{0}
$$

this additional constraint involves the mean value of the two constant vectors to which $\mathbf{V}^{i j}$ tends at infinity, and it completes (28) which only gives the difference $\mathbf{V}^{i j+}-\mathbf{V}^{i j-}$.

Remark 5. In the case of a crack we have $\operatorname{vol}(\mathcal{D})=0$ and $\mathcal{D}=\partial \mathcal{D}$. Thus, if we do not take into account the possibility of interpenetration of the lips of the crack, the elementary problems remain essentially the same as in the case of a hole, it suffices to read accordingly the boundary condition. Of course, it is unnecessary (and even meaningless) to extend the fields in $\mathcal{D}$ since the interior of $\mathcal{D}$ is empty. The situation is slightly different when we want to forbid the interpenetration of the lips. In such a case, the boundary condition in the real problem must be changed into unilateral contact conditions. Accordingly, if we assume that the contact takes place without friction, the contact conditions read as

$$
\boldsymbol{\sigma}^{\epsilon} \mathbf{n} \wedge \mathbf{n}=\mathbf{0}, \quad \llbracket \mathbf{u}^{\epsilon} \rrbracket \cdot \mathbf{n} \geq 0, \quad \boldsymbol{\sigma}^{\epsilon} \mathbf{n} \cdot \mathbf{n} \leq 0, \quad \boldsymbol{\sigma}^{\epsilon} \mathbf{n} \cdot \mathbf{n} \llbracket \mathbf{u}^{\epsilon} \rrbracket \cdot \mathbf{n}=0 .
$$

In terms of the inner fields $\hat{\mathbf{v}}$ and $\hat{\boldsymbol{\tau}}$ they read as

$$
\left(\hat{\boldsymbol{\tau}}+\boldsymbol{\sigma}^{0}\right) \mathbf{n} \wedge \mathbf{n}=\mathbf{0}, \quad \llbracket \hat{\mathbf{v}} \rrbracket \cdot \mathbf{n} \geq 0, \quad\left(\hat{\boldsymbol{\tau}}+\boldsymbol{\sigma}^{0}\right) \mathbf{n} \cdot \mathbf{n} \leq 0, \quad\left(\hat{\boldsymbol{\tau}}+\boldsymbol{\sigma}^{0}\right) \mathbf{n} \cdot \mathbf{n} \llbracket \hat{\mathbf{v}} \rrbracket \cdot \mathbf{n}=0 \quad \text { on } \mathcal{D} .
$$


Therefore, we lose in general the linearity property and the order 0 inner problem cannot be decomposed into 6 elementary problems. However, this decomposition remains possible when the geometry of the crack is simple, see the examples in the last section.

The six universal problems will be studied in more detail in the next sections. At the present time, we will assume that they are solved, which implies that $\mathbf{v}^{1}$ and $\tau^{0}$ are now known, except for the arbitrary field $\check{\mathbf{v}}$ entering the expression of $\hat{\mathbf{v}}$. In fact, $\check{\mathbf{v}}$ will be determined once $\mathbf{u}^{1}$ is known. Indeed, the matching condition (13) becomes, in view of (22) , (26) and (30),

$$
\mathbf{u}^{1 \pm}\left(\mathbf{x}^{\prime}\right)=\sigma_{i j}^{0}\left(0, \mathbf{x}^{\prime}\right) \mathbf{V}^{i j \pm}+\check{\mathbf{v}}\left(\mathbf{x}^{\prime}\right) .
$$

By addition of the two limits, (31) yields the desired relation between $\check{\mathbf{v}}$ and $\hat{\mathbf{u}}$ :

$$
2 \check{\mathbf{v}}\left(\mathbf{x}^{\prime}\right)=\mathbf{u}^{1+}\left(\mathbf{x}^{\prime}\right)+\mathbf{u}^{1-}\left(\mathbf{x}^{\prime}\right) .
$$

In other words, $\check{\mathbf{v}}$ is the mean value of $\mathbf{u}^{1}$ on $\boldsymbol{\Gamma}$.

3.5. Construction of the order 1 outer problem. This subsection is devoted to the construction of the problem giving $\mathbf{u}^{1}$ and $\boldsymbol{\sigma}^{1}$. We already know that $\mathbf{u}^{1}$ and $\boldsymbol{\sigma}^{1}$ must satisfy the order 1 outer equilibrium equations, outer constitutive relations and outer boundary conditions, i.e.

$$
\begin{aligned}
& \operatorname{div} \boldsymbol{\sigma}^{1}=\mathbf{0} \quad \text { in } \quad \boldsymbol{\Omega} \backslash \boldsymbol{\Gamma}, \quad \boldsymbol{\sigma}^{1}=\mathrm{A} \varepsilon\left(\mathbf{u}^{1}\right) \quad \text { in } \quad \boldsymbol{\Omega} \backslash \boldsymbol{\Gamma} \\
& \boldsymbol{\sigma}^{1} \mathbf{n}=\mathbf{0} \quad \text { on } \quad \partial_{F} \boldsymbol{\Omega}, \quad \mathbf{u}^{1}=\mathbf{0} \quad \text { on } \partial_{D} \boldsymbol{\Omega}
\end{aligned}
$$

What is missing are the jump conditions across $\boldsymbol{\Gamma}$. We immediately deduce from (33) that

$$
\llbracket \mathbf{u}^{1} \rrbracket\left(\mathbf{x}^{\prime}\right)=\sigma_{i j}^{0}\left(0, \mathbf{x}^{\prime}\right)\left(\mathbf{V}^{i j+}-\mathbf{V}^{i j-}\right) .
$$

To obtain the jump condition satisfied by $\boldsymbol{\sigma}^{1} \mathbf{e}_{1}$, we first integrate the order 1 stress matching condition (14) over $\mathbf{P}$ and obtain

$$
\boldsymbol{\sigma}^{1 \pm}\left(\mathbf{x}^{\prime}\right)=\lim _{y_{1} \rightarrow \pm \infty}\left(\int_{\mathbf{P}} \boldsymbol{\tau}^{1}\left(\mathbf{x}^{\prime}, y_{1}, \mathbf{y}^{\prime}\right) d \mathbf{y}^{\prime}-y_{1} \frac{\partial \boldsymbol{\sigma}^{0}}{\partial x_{1}}\left(0, \mathbf{x}^{\prime}\right)\right) .
$$

Further, using (23), the order 0 inner equilibrium equations, that is $\operatorname{div}_{\mathbf{y}} \boldsymbol{\tau}^{1}+\mathbf{d i v}_{\mathbf{x}} \boldsymbol{\tau}^{0}=0$, can read as

$$
\frac{\partial \tau_{i j}^{1}}{\partial y_{j}}\left(\mathbf{x}^{\prime}, \mathbf{y}\right)+\frac{\partial \sigma_{i \alpha}^{0}}{\partial x_{\alpha}}\left(0, \mathbf{x}^{\prime}\right)+\frac{\partial \hat{\tau}_{i \alpha}}{\partial x_{\alpha}}\left(\mathbf{x}^{\prime}, \mathbf{y}\right)=0 \quad \text { in } \quad \boldsymbol{\Gamma} \times \mathbf{Y}
$$

once we have extended $\boldsymbol{\tau}^{0}$ and $\boldsymbol{\tau}^{1}$ by $\mathbf{0}$ in $\mathcal{D}$. Integrating first over $\left(-y_{1},+y_{1}\right) \times \mathbf{P}$ and then passing to the limit when $y_{1}$ goes to infinity lead to

$$
\frac{\partial}{\partial x_{\alpha}} \int_{\mathbf{Y}} \hat{\tau}_{i \alpha}\left(\mathbf{x}^{\prime}, \mathbf{y}\right) d \mathbf{y}=-\lim _{y_{1} \rightarrow \infty}\left(\int_{\mathbf{P}}\left(\tau_{i 1}^{1}\left(\mathbf{x}^{\prime}, y_{1}, \mathbf{y}^{\prime}\right) d \mathbf{y}^{\prime}-\tau_{i 1}^{1}\left(\mathbf{x}^{\prime},-y_{1}, \mathbf{y}^{\prime}\right)\right) d \mathbf{y}^{\prime}+2 y_{1} \frac{\partial \sigma_{i \alpha}^{0}}{\partial x_{\alpha}}\left(0, \mathbf{x}^{\prime}\right)\right) .
$$

In the derivation of (39) we have used the periodic conditions satisfied by $\boldsymbol{\tau}^{1}$ on $\mathbb{R} \times \partial \mathbf{P}$. But, since $\partial \sigma_{i 1}^{0} / \partial x_{1}=-\partial \sigma_{i \alpha}^{0} / \partial x_{\alpha}$, inserting (37) into (39) gives

$$
\llbracket \sigma_{i 1}^{1} \rrbracket\left(\mathbf{x}^{\prime}\right)=-\frac{\partial}{\partial x_{\alpha}} \int_{\mathbf{Y}} \hat{\tau}_{i \alpha}\left(\mathbf{x}^{\prime}, \mathbf{y}\right) d \mathbf{y} .
$$


Finally, using (27) leads to

$$
\llbracket \sigma_{k 1}^{1} \rrbracket\left(\mathbf{x}^{\prime}\right)=-\frac{\partial \sigma_{i j}^{0}}{\partial x_{\alpha}}\left(0, \mathbf{x}^{\prime}\right) \int_{\mathbf{Y}} T_{k \alpha}^{i j}(\mathbf{y}) d \mathbf{y}
$$

where $\mathbf{T}^{i j}$ is extended to $\mathcal{D}$ by $(29)$.

Let us remark that the jump of $\sigma_{11}^{1}$ always vanishes. Indeed, we have $\operatorname{div} \mathbf{T}^{i j}=\mathbf{0}$ in $\mathbf{Y}$. Integrating over $\mathbf{P}$ at a given $y_{1}$ and using the periodic condition on $\partial \mathbf{P}$ give

$$
\frac{\partial}{\partial y_{1}} \int_{\mathbf{P}} T_{k 1}^{i j}\left(y_{1}, \mathbf{y}^{\prime}\right) d \mathbf{y}^{\prime}=0, \quad \forall y_{1} \in \mathbb{R}, \forall k \in\{1,2,3\}
$$

Hence, using the vanishing of $\mathbf{T}^{i j}$ at infinity, we obtain $\int_{\mathbf{P}} T_{k 1}^{i j}\left(y_{1}, \mathbf{y}^{\prime}\right) d \mathbf{y}^{\prime}=0$ for all $y_{1}$ and all $k$. Integrating in $y_{1}$ gives

$$
\int_{\mathbf{Y}} T_{k 1}^{i j}(\mathbf{y}) d \mathbf{y}=\int_{\mathbf{Y}} T_{1 k}^{i j}(\mathbf{y}) d \mathbf{y}=0, \quad \forall i, j, k \in\{1,2,3\} .
$$

Inserting in (41) gives the desired result. We have thus obtained the following fundamental result

Proposition 3. The order 1 outer displacement and stress fields $\mathbf{u}^{1}$ and $\boldsymbol{\sigma}^{1}$ have to satisfy the following jump conditions across $\boldsymbol{\Gamma}$, expressed in terms of the order 0 outer stress field $\boldsymbol{\sigma}^{0}$ and its derivatives on $\boldsymbol{\Gamma}: \forall k \in\{1,2,3\}$ and $\forall \alpha \in\{2,3\}$,

$$
\llbracket u_{k}^{1} \rrbracket\left(\mathbf{x}^{\prime}\right)=\mathrm{B}_{k i j} \sigma_{i j}^{0}\left(0, \mathbf{x}^{\prime}\right), \quad \llbracket \sigma_{\alpha 1}^{1} \rrbracket\left(\mathbf{x}^{\prime}\right)=-\mathrm{C}_{\alpha \beta i j} \frac{\partial \sigma_{i j}^{0}}{\partial x_{\beta}}\left(0, \mathbf{x}^{\prime}\right),
$$

where the third order tensor $\mathrm{B}$ and the fourth order tensor $\mathrm{C}$ are related to the solutions of the six elementary inner problems by

$$
\mathrm{B}_{k i j}=V_{k}^{i j+}-V_{k}^{i j-}, \quad \mathrm{C}_{\alpha \beta i j}=\int_{\mathbf{Y}} T_{\alpha \beta}^{i j}(\mathbf{y}) d \mathbf{y} .
$$

Remark 6. Those jump conditions across $\boldsymbol{\Gamma}$ are of Ventcel's type when the tensor $\mathbf{C}$ is not zero. That means that, in general, the influence of the holes cannot be identified with a surface density of springs as it is usually assumed in simplified models, cf Rose (1987), see also the examples in the last section. Indeed, a surface density of springs give rise to Robin's type transmission conditions, i.e. the jump conditions can read as

$$
\llbracket u_{i}^{1} \rrbracket=B_{i j} \sigma_{j 1}^{0}, \quad \llbracket \sigma_{i 1}^{1} \rrbracket=0 \quad \forall i \in\{1,2,3\} .
$$

Thus, in (43) the jump of the displacement depends on all the components of the stress tensor $\boldsymbol{\sigma}^{0}$ at the interface while it depends only of the components of the stress vector $\boldsymbol{\sigma}^{0} \mathbf{e}_{1}$ in Robin's condition; the stress vector is discontinuous in (43) while it is continuous in Robin's condition.

Note, however, that (43) are not exactly the jump conditions which appear when the material in the layer is stiff and constitute the "true" Ventcel's conditions, cf Bessoud et al. (2008). 
Indeed, in such a case, $u^{0}$ and $\sigma^{0}$ are discontinuous and the jump conditions read as

$$
\llbracket u_{k}^{0} \rrbracket=\mathrm{B}_{k i j} \overline{\sigma_{i j}^{0}}, \quad \llbracket \sigma_{\alpha 1}^{0} \rrbracket=-\mathrm{C}_{\alpha \beta i j} \frac{\partial \overline{\sigma_{i j}^{0}}}{\partial x_{\beta}}
$$

where $\bar{f}=\left(f^{+}+f^{-}\right) / 2$.

To complete the construction of the order 1 outer problem, we should carefully analyze the behavior of the fields $\mathbf{u}^{1}$ and $\boldsymbol{\sigma}^{1}$ near the boundary $\partial \boldsymbol{\Gamma}$. First we can see from (41) that there generally exists a misfit between the vanishing of the stress vector on the boundary $\left(\boldsymbol{\sigma}^{1} \mathbf{n}=\mathbf{0}\right.$ on $\left.\partial_{F} \boldsymbol{\Omega}\right)$ and the jump of $\boldsymbol{\sigma}^{1} \mathbf{e}_{1}$ on $\boldsymbol{\Gamma}$ because the limit of the latter one will not be, in general, equal to 0 when $\mathbf{x}^{\prime}$ goes to $\partial \boldsymbol{\Omega}$. That shows that a second boundary layer effect exists in the neighborhood of $\partial \boldsymbol{\Gamma}$ which requires to consider a third type of asymptotic expansion of the solution in this zone. A careful analysis should also show that this misfit leads to the existence of a linear density of forces on $\partial \boldsymbol{\Gamma}$ for the order 1 outer problem which induces singularities in the fields $\mathbf{u}^{1}$ and $\boldsymbol{\sigma}^{1}$. However this part of the analysis is outside the scope of the present paper and the interested reader can refer to Abdelmoula and Marigo (2000) for such an analysis. Accordingly, we consider that the construction of the order 1 outer problem is closed and we will focus on the analysis of the jump conditions (43) and on the involved coefficients (44) in the last section.

\section{The efFective Damage tensor and the EFfeCtive transmission CONDitions}

4.1. The effective damage compliance tensor associated with the holed cell. Let us define the fourth order tensor $\mathrm{D}$ by

$$
\mathrm{D}_{i j k l}=\int_{\mathbf{Y}} \mathrm{A}^{-1} \mathbf{T}^{i j} \cdot \mathbf{T}^{k l} d \mathbf{y}
$$

where the $\mathbf{T}^{i j}$ 's have been extended to $\mathcal{D}$ by $(29)$ and $A^{-1}$ denotes the inverse of $A$, i.e. the compliance tensor of the material. D can be also expressed in terms of the $\mathbf{V}^{i j}$ 's without using their extensions, namely

$$
\mathrm{D}_{i j k l}=\int_{\mathbf{Y} \backslash \mathcal{D}} \mathrm{A} \varepsilon\left(\mathbf{V}^{i j}\right) \cdot \varepsilon\left(\mathbf{V}^{k l}\right) d \mathbf{y}+\mathrm{A}_{i j k l}^{-1} \operatorname{vol}(\mathcal{D}) .
$$

Note that $\mathrm{D}$ has the dimension of the inverse of a pressure, like a compliance tensor, since $\mathbf{y}$ and $\mathbf{T}^{i j}$ are dimensionless. Let $\boldsymbol{\sigma}$ be a stress tensor corresponding to the applied loading to the cell at infinity. Then, $\sigma_{i j} \mathbf{T}^{i j}$ represents the difference between the stress field which holds in the holed cell and the constant stress field $\boldsymbol{\sigma}$ which would hold in the sound cell. Therefore, $\mathrm{D} \boldsymbol{\sigma} \cdot \boldsymbol{\sigma}$ can be seen as a measure of the increase of elastic energy due to the hole in the holed cell (which becomes more compliant) submitted to $\boldsymbol{\sigma}$ at infinity. (Note also that $\mathbf{T}^{i j}=\mathbf{0}$ and hence $\mathrm{D}=0$ when there is no hole in the cell.) Accordingly, $\mathrm{D}$ can be considered as the effective damage compliance tensor associated with the holed cell. 
It is also useful to give a variational definition of the damage tensor so that to compare the effects of different defects. From the classical theorem of the minimum of the complementary energy in linear elasticity, we get

$$
\mathrm{D} \boldsymbol{\sigma} \cdot \boldsymbol{\sigma}=\min _{\mathbf{T} \in \mathcal{S}(\boldsymbol{\sigma})} \int_{\mathbf{Y}} \mathrm{A}^{-1} \mathbf{T} \cdot \mathbf{T} d \mathbf{y}
$$

where $\mathcal{S}(\boldsymbol{\sigma})$ denotes the set of statically admissible stress fields, i.e.

$$
\mathcal{S}(\boldsymbol{\sigma})=\left\{\mathbf{T}: \mathbf{T} \mathbf{P} \text {-periodic }, \quad \lim _{\left|y_{1}\right| \rightarrow \infty} \mathbf{T}(\mathbf{y})=0, \quad \operatorname{div} \mathbf{T}=\mathbf{0} \text { in } \mathbf{Y}, \quad \mathbf{T}=-\boldsymbol{\sigma} \text { in } \mathcal{D}\right\}
$$

(Of course, the minimizer is $\mathbf{T}=\sigma_{i j} \mathbf{T}^{i j}$.) We immediately deduce from its definition (46) that D admits some symmetry and positivity properties. Specifically, we have

Proposition 4. The damage tensor $\mathrm{D}$ admits the classical symmetry properties of a compliance tensor, namely

$$
\mathrm{D}_{i j k l}=\mathrm{D}_{j i k l}=\mathrm{D}_{k l i j}, \quad \forall i, j, k, l \in\{1,2,3\} .
$$

Moreover, $\mathrm{D}$ is non negative and even $\mathrm{D}$ is positive if the defect is a hole, i.e. if $\operatorname{vol}(\mathcal{D})>0$. $\mathrm{D}$ is non definite only when the defect is a "planar" crack (that is an union of cracks whose unit current normal is parallel to a fix direction) or an "antiplanar" crack (that is an union of cracks whose unit current normal remains orthogonal to a fix direction).

Furthermore $\mathrm{D}$ is an increasing function of the size of the defect, i.e. $\mathrm{D}(\mathcal{D}) \geq \mathrm{D}\left(\mathcal{D}^{\prime}\right)$ if $\mathcal{D} \supset \mathcal{D}^{\prime}$.

Proof. The symmetry of $D$ is a direct consequence of its definition (46), of the fact that $\mathbf{T}^{i j}=\mathbf{T}^{i j}$ by construction and of the symmetry of $\mathrm{A}^{-1}$. Moreover, by virtue of the positivity of $A^{-1}$, we deduce also from (46) that $D$ is non negative and even we have by virtue of (47) the following lower bound

$$
\mathrm{D} \boldsymbol{\sigma} \cdot \boldsymbol{\sigma} \geq \operatorname{vol}(\mathcal{D}) \mathrm{A}^{-1} \boldsymbol{\sigma} \cdot \boldsymbol{\sigma} \geq 0, \quad \forall \boldsymbol{\sigma}
$$

Therefore $\mathrm{D}$ is positive if $\operatorname{vol}(\mathcal{D})>0$.

Let us now consider the case of a crack, i.e. when $\operatorname{vol}(\mathcal{D})=0$ and $\mathcal{D}=\partial \mathcal{D}$. $\mathrm{D}$ is non definite if and only if there exists $\boldsymbol{\sigma} \neq \mathbf{0}$ such that $\mathrm{D} \boldsymbol{\sigma} \cdot \boldsymbol{\sigma}=0$. Assume that such a tensor $\boldsymbol{\sigma}$ exists. By virtue of (46), D $\boldsymbol{\sigma} \cdot \boldsymbol{\sigma}=0$ is equivalent to $\sigma_{i j} \mathbf{T}^{i j}=\mathbf{0}$ in $\mathbf{Y} \backslash \mathcal{D}$. Using (28), it is equivalent to $\boldsymbol{\sigma} \mathbf{n}=\mathbf{0}$ on $\mathcal{D}$. Therefore $\mathbf{n}(\mathbf{y})$ must be an eigenvector of $\boldsymbol{\sigma}$ associated with a necessarily 0 eigenvalue of $\boldsymbol{\sigma}$ for every $\mathbf{y} \in \mathcal{D}$. Since $\boldsymbol{\sigma} \neq \mathbf{0}, \boldsymbol{\sigma}$ has necessarily a non zero eigenvalue. Let $\mathcal{N}$ be the eigenspace of $\boldsymbol{\sigma}$ associated with its 0 eigenvalue, we have $1 \leq \operatorname{dim} \mathcal{N} \leq 2$. Since $\mathbf{n}(\mathbf{y})$ must belong to $\mathcal{N}$ for every $\mathbf{y}$ on $\mathcal{D}$, we have to consider two cases:

(1) If $\operatorname{dim} \mathcal{N}=1$, then $\mathbf{n}(\mathbf{y})$ is parallel to a fix direction, say $\boldsymbol{\nu}$. That means that $\mathbf{n}(\mathbf{y})= \pm \boldsymbol{\nu}$ for every $\mathbf{y} \in \mathcal{D}$, the crack is planar in the sense given in the statement of the Proposition. (Note that $\mathcal{D}$ can correspond to a family of parallel planar connected cracks.) Conversely, if the crack is planar with $\boldsymbol{\nu}$ as normal vector, then taking $\boldsymbol{\sigma}=\mathbf{t} \otimes \mathbf{t}$ with $\mathbf{t} \cdot \boldsymbol{\nu}=0$, we have $\boldsymbol{\sigma} \boldsymbol{\nu}=\mathbf{0}$ and hence $\mathrm{D} \boldsymbol{\sigma} \cdot \boldsymbol{\sigma}=0$. 
(2) If $\operatorname{dim} \mathcal{N}=2$, then $\mathbf{n}(\mathbf{y})$ must belong to $\mathcal{N}$ and hence be orthogonal to a fix direction, say $\boldsymbol{\nu}$. That means that $\mathbf{n}(\mathbf{y}) \cdot \boldsymbol{\nu}=0$ for every $\mathbf{y} \in \mathcal{D}$, the crack is anti-planar in the sense given in the statement of the Proposition. Conversely, if the crack is anti-planar with $\boldsymbol{\nu}$ as a vector orthogonal to every $\mathbf{n}(\mathbf{y})$, then taking $\boldsymbol{\sigma}=\boldsymbol{\nu} \otimes \boldsymbol{\nu}$, we get $\boldsymbol{\sigma n}(\mathbf{y})=\mathbf{0}$ for every $\mathbf{y} \in \mathcal{D}$ and hence $\mathrm{D} \boldsymbol{\sigma} \cdot \boldsymbol{\sigma}=0$.

Let $\mathcal{D}$ and $\mathcal{D}^{\prime}$ be two defects with $\mathcal{D} \supset \mathcal{D}^{\prime}$. Let us denote by $\mathrm{D}$ and $\mathrm{D}^{\prime}$ the associated damage tensors and by $\mathcal{S}(\boldsymbol{\sigma})$ and $\mathcal{S}^{\prime}(\boldsymbol{\sigma})$ the associated set of statically stress fields for the same "loading" $\boldsymbol{\sigma}$, cf (49). Since $\mathcal{S}(\boldsymbol{\sigma}) \subset \mathcal{S}^{\prime}(\boldsymbol{\sigma})$, we have $\mathrm{D} \boldsymbol{\sigma} \cdot \boldsymbol{\sigma}=\min _{\mathcal{S}(\boldsymbol{\sigma})} \int_{\mathbf{Y}} \mathrm{A}^{-1} \mathbf{T} \cdot \mathbf{T} d \mathbf{y} \geq \min _{\mathcal{S}^{\prime}(\boldsymbol{\sigma})} \int_{\mathbf{Y}} \mathrm{A}^{-1} \mathbf{T}$. $\mathbf{T} d \mathbf{y}=\mathrm{D}^{\prime} \boldsymbol{\sigma} \cdot \boldsymbol{\sigma}$ and hence $\mathrm{D} \geq \mathrm{D}^{\prime}$.

By virtue of (50), D has, at most, 21 independent coefficients. This number is smaller when the material, the cell and the hole possess symmetries. The reader interested by a general discussion on the symmetries of effective elastic tensors in the framework of the homogenization theory of periodic media can refer to Léné (1984), Suquet (1987) or Michel et al. (1999). We will only consider particular cases in the examples below.

4.2. The relationships between the tensors B, C and D. By construction the tensors B and $C$ given by (44) have the following symmetries:

$$
\mathrm{B}_{k i j}=\mathrm{B}_{k j i}, \quad \mathrm{C}_{\alpha \beta i j}=\mathrm{C}_{\beta \alpha i j}=\mathrm{C}_{\alpha \beta j i}, \quad \forall i, j, k \in\{1,2,3\}, \quad \forall \alpha, \beta \in\{2,3\} .
$$

Accordingly, there exist at most $3 \times 6=18$ independent coefficients for B and also $3 \times 6=18$ independent coefficients for $C$. It turns out that these 36 coefficients can be expressed in terms of the coefficients of the damage compliance tensor D of the holed cell and of the stiffness tensor A of the material. Before establishing these relations, let us introduce the

Definition 1. Let $\mathrm{P}$ be the projection onto the in-plane symmetric second order tensors, that is the symmetric fourth order tensor which associate to $\boldsymbol{\sigma}=\sigma_{i j} \mathbf{e}_{i} \otimes \mathbf{e}_{j}$ the tensor $\mathrm{P} \boldsymbol{\sigma}$ defined by

$$
\mathrm{P} \boldsymbol{\sigma}=\sigma_{\alpha \beta} \mathbf{e}_{\alpha} \otimes \mathbf{e}_{\beta},
$$

recalling that the Latin indices run from 1 to 3 and the Greek indices from 2 to 3.

Specifically, we have

Proposition 5. The components of $\mathrm{B}$ are related to those of $\mathrm{D}$ by

$$
\mathrm{B}_{1 i j}=\mathrm{D}_{11 i j}-\mathrm{A}_{11 \alpha \beta}^{-1} \mathrm{~A}_{\alpha \beta \gamma \delta}^{\mathrm{P}} \mathrm{D}_{\gamma \delta i j}, \quad \mathrm{~B}_{\eta i j}=2 \mathrm{D}_{\eta 1 i j}-2 \mathrm{~A}_{\eta 1 \alpha \beta}^{-1} \mathrm{~A}_{\alpha \beta \gamma \delta}^{\mathrm{P}} \mathrm{D}_{\gamma \delta i j} \text {, }
$$

whereas the components of $\mathrm{C}$ are related to those of $\mathrm{D}$ by

$$
\mathrm{C}_{\alpha \beta i j}=-\mathrm{A}_{\alpha \beta \gamma \delta}^{\mathrm{P}} \mathrm{D}_{\gamma \delta i j} \text {, }
$$

where $\mathrm{A}^{\mathrm{P}}$ denotes the "in-plane" stiffness tensor, i.e. the symmetric fourth order tensor such that $\mathrm{A}^{\mathrm{P} P A}{ }^{-1} \mathrm{P}=\mathrm{P}$. 
Proof. Let $\boldsymbol{\sigma}$ and $\boldsymbol{\sigma}^{*}$ be two symmetric second order tensors. Let $\boldsymbol{\tau}$ and $\boldsymbol{\tau}^{*}$ be the associated stress tensor fields, i.e. $\boldsymbol{\tau}(\mathbf{y})=\boldsymbol{\sigma}+\sigma_{i j} \mathbf{T}^{i j}(\mathbf{y})$ and $\boldsymbol{\tau}^{*}(\mathbf{y})=\boldsymbol{\sigma}^{*}+\sigma_{i j}^{*} \mathbf{T}^{i j}(\mathbf{y})$, which are extended to $\mathbf{0}$ in $\mathcal{D}$. Let $\mathbf{v}$ and $\mathbf{v}^{*}$ be the associated displacement fields, i.e. $\mathbf{v}(\mathbf{y})=\sigma_{i j} \mathbf{V}^{i j}(\mathbf{y})$ and $\mathbf{v}^{*}(\mathbf{y})=\sigma_{i j}^{*} \mathbf{V}^{i j}(\mathbf{y})$. Let us compute $\int_{\mathbf{Y} \backslash \mathcal{D}} \tau^{*} \cdot \varepsilon(\mathbf{v}) d \mathbf{y}$ by two ways.

First, using (28) and (30), we have

$$
\begin{aligned}
\int_{\mathbf{Y} \backslash \mathcal{D}} \boldsymbol{\tau}^{*} \cdot \boldsymbol{\varepsilon}(\mathbf{v}) d \mathbf{y} & =-\int_{\mathbf{Y} \backslash \mathcal{D}} \operatorname{div} \boldsymbol{\tau}^{*} \cdot \mathbf{v} d \mathbf{y}+\int_{\partial(\mathbf{Y} \backslash \mathcal{D})} \boldsymbol{\tau}^{*} \mathbf{n} \cdot \mathbf{v} d S \\
& =\boldsymbol{\sigma}^{*} \mathbf{e}_{1} \cdot\left(\mathbf{V}^{i j+}-\mathbf{V}^{i j-}\right) \sigma_{i j} .
\end{aligned}
$$

Therefore, by virtue of (44), we get

$$
\int_{\mathbf{Y} \backslash \mathcal{D}} \tau^{*} \cdot \varepsilon(\mathbf{v}) d \mathbf{y}=\mathrm{B}_{k i j} \sigma_{k 1}^{*} \sigma_{i j}
$$

Second, using (28) and (47), we get

$$
\begin{aligned}
\int_{\mathbf{Y} \backslash \mathcal{D}} \tau^{*} \cdot \boldsymbol{\varepsilon}(\mathbf{v}) d \mathbf{y} & =\boldsymbol{\sigma}^{*} \cdot \int_{\mathbf{Y} \backslash \mathcal{D}} \boldsymbol{\varepsilon}(\mathbf{v}) d \mathbf{y}+\int_{\mathbf{Y} \backslash \mathcal{D}} \mathrm{A} \boldsymbol{\varepsilon}\left(\mathbf{v}^{*}\right) \cdot \boldsymbol{\varepsilon}(\mathbf{v}) d \mathbf{y} \\
& =\mathrm{A}^{-1} \boldsymbol{\sigma}^{*} \cdot \int_{\mathbf{Y} \backslash \mathcal{D}} \mathbf{T}^{i j} \sigma_{i j} d \mathbf{y}+\int_{\mathbf{Y} \backslash \mathcal{D}} \mathrm{A} \varepsilon\left(\mathbf{V}^{i j} \sigma_{i j}^{*}\right) \cdot \boldsymbol{\varepsilon}\left(\mathbf{V}^{k l} \sigma_{k l}\right) d \mathbf{y} \\
& =\mathrm{A}^{-1} \boldsymbol{\sigma}^{*} \cdot \int_{\mathbf{Y} \backslash \mathcal{D}} \mathbf{T}^{i j} \sigma_{i j} d \mathbf{y}-\mathrm{A}^{-1} \boldsymbol{\sigma}^{*} \cdot \boldsymbol{\sigma} \operatorname{vol}(\mathcal{D})+\mathrm{D} \boldsymbol{\sigma}^{*} \cdot \boldsymbol{\sigma} \\
& =\mathrm{A}^{-1} \boldsymbol{\sigma}^{*} \cdot \int_{\mathbf{Y}} \mathbf{T}^{i j} \sigma_{i j} d \mathbf{y}+\mathrm{D} \boldsymbol{\sigma}^{*} \cdot \boldsymbol{\sigma} .
\end{aligned}
$$

Therefore, by virtue of (44), we have also

$$
\int_{\mathbf{Y} \backslash \mathcal{D}} \boldsymbol{\tau}^{*} \cdot \varepsilon(\mathbf{v}) d \mathbf{y}=\mathrm{A}_{\alpha \beta k l}^{-1} \mathrm{C}_{\alpha \beta i j} \sigma_{k l}^{*} \sigma_{i j}+\mathrm{D}_{i j k l} \sigma_{k l}^{*} \sigma_{i j} .
$$

Comparing (56) and (57), which hold for all $\boldsymbol{\sigma}$ and $\boldsymbol{\sigma}^{*}$, leads to

$$
\mathrm{B}_{k i j} \sigma_{k 1}^{*}=\left(\mathrm{A}_{\alpha \beta k l}^{-1} \mathrm{C}_{\alpha \beta i j}+\mathrm{D}_{i j k l}\right) \sigma_{k l}^{*}, \quad \forall \boldsymbol{\sigma}^{*} .
$$

Taking first for $\boldsymbol{\sigma}^{*}$ an in-plane tensor, i.e. $\boldsymbol{\sigma}^{*}$ such that $\mathrm{P} \boldsymbol{\sigma}^{*}=\boldsymbol{\sigma}^{*}$, the left-hand side of (58) vanishes and we obtain $\mathrm{A}_{\alpha \beta \gamma \delta}^{-1} \mathrm{C}_{\gamma \delta i j}=-\mathrm{D}_{\alpha \beta i j}$. In an intrinsic form, since $\mathrm{PC}=\mathrm{C}$, that reads as $P A^{-1} P C=-P D$. Multiplying on the left by $A^{P}$ and using $A^{P} P=P A^{P}=A^{P}$, we get $A^{P} P A^{-1} P C=$ $-A^{P} D$. Using the definition of $A^{P}$, we obtain $C=-A^{P} D$, that is (55). Taking then $\boldsymbol{\sigma}=\mathbf{e}_{1} \otimes \mathbf{e}_{1}$ in (58) we get $\mathrm{B}_{1 i j}=\mathrm{A}_{11 \alpha \beta}^{-1} \mathrm{C}_{\alpha \beta i j}+\mathrm{D}_{11 i j}$, which can read as $\mathrm{B}_{1 i j}=\left(\mathrm{A}^{-1} \mathrm{C}+\mathrm{D}\right)_{11 i j}$. Using (55) we obtain $\mathrm{B}_{1 i j}=\left(\mathrm{D}-\mathrm{A}^{-1} \mathrm{~A}^{\mathrm{P} D}\right)_{11 i j}$, that is (54a). Finally, taking $\boldsymbol{\sigma}=2 \mathbf{e}_{\eta} \otimes_{s} \mathbf{e}_{1}$ in (58) and using (55), we get (54b).

Of course, the symmetries of $A$ and $D$ are directly transmitted to $B$ and $C$. On the other hand, the general positivity properties of $B$ and $C$ need a more careful analysis. It is outside the scope of this paper. 
4.3. Examples. We consider three "typical" examples: one where the defect is a hole, the two other where the defect is a crack. In each case, the material is isotropic. Accordingly, the compliance tensor $A^{-1}$ and the in-plane stiffness tensor $A^{P}$ of the material are given by

$$
\mathrm{A}^{-1}=\frac{1+\nu}{E} \mathrm{I}-\frac{\nu}{E} \mathbf{I} \otimes \mathbf{I}, \quad \mathrm{A}^{\mathrm{P}}=\frac{E}{1+\nu}\left(\mathrm{P}+\frac{\nu}{1-\nu} \mathrm{PI} \otimes \mathrm{I} \mathbf{I}\right),
$$

where I and I are respectively the fourth order and the second order identity tensors, and $E$ and $\nu$ are the Young modulus and the Poisson ratio of the material. Moreover, the dependence of D on $E$ is explicit and $\mathrm{D}$ can be read as

$$
\mathrm{D}=\frac{1}{E} \hat{\mathrm{D}}(\nu, \mathbf{a}, \mathbf{b}, \mathcal{D})
$$

where $\hat{D}$ is a dimensionless fourth order tensor whose components depend on the geometrical parameters of the holed cell and on the Poisson ratio.

EXAMPLE 1: (An hexagonal array of spherical holes) Let $\ell=3^{-1 / 4} \sqrt{2}, \mathbf{a}=\ell \mathbf{e}_{2}, \mathbf{b}=$ $\ell\left(\mathbf{e}_{2}+\sqrt{3} \mathbf{e}_{3}\right) / 2$ and $\mathcal{D}$ be the ball of center $\mathbf{0}$ and radius $R<\ell / 2$. Thus the defect is a spherical hole of radius $R$ and we can choose for $\mathbf{P}$ a perfect hexagon with center $\mathbf{0}$, see Figure 4 . Since
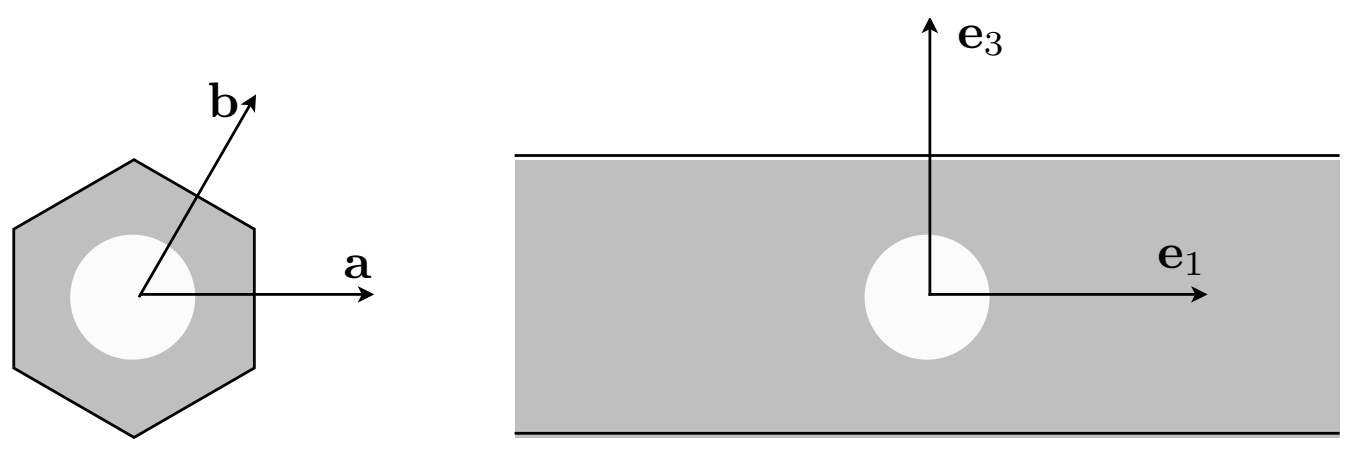

Figure 4. Case when the defect is a spherical hole

the material is isotropic, we can use the results of Léné (1984) to obtain that D is a positive transversely isotropic fourth order tensor with axis $\mathbf{e}_{1}$. Therefore, D can be written

$$
\mathrm{D} \boldsymbol{\sigma} \cdot \boldsymbol{\sigma}=\frac{d_{L}}{E} \sigma_{11}^{2}+\frac{2 \delta_{L}}{E} \sigma_{11} \sigma_{\alpha \alpha}+\frac{\delta_{T}}{E} \sigma_{\alpha \alpha}^{2}+\frac{2 d_{T}}{E} \sigma_{\alpha \beta} \sigma_{\alpha \beta}+\frac{4 d_{S}}{E} \sigma_{1 \alpha} \sigma_{1 \alpha}
$$

where the five dimensionless coefficients $d_{L}, d_{T}, d_{S}, \delta_{T}$ and $\delta_{L}$ are functions of $R$ and $\nu$ which satisfy the following inequalities:

$$
d_{L}>0, \quad d_{T}>0, \quad d_{S}>0, \quad \delta_{T}+d_{T}>0, \quad\left(\delta_{T}+d_{T}\right) d_{L}>\delta_{L}^{2} .
$$


Inserting (61) into (54)-(55) and using (59) give the tensors B and C. The details of the calculations are left to the reader. We finally obtain

$$
\left(\begin{array}{l|l}
\mathrm{B}_{111}=\frac{d_{L}}{E}+\frac{2 \nu}{1-\nu} \frac{\delta_{L}}{E} & \mathrm{C}_{2211}=\mathrm{C}_{3311}=-\frac{\delta_{L}}{1-\nu} \\
\mathrm{B}_{212}=\mathrm{B}_{313}=\frac{2 d_{S}}{E} & \mathrm{C}_{2222}=\mathrm{C}_{3333}=-\frac{\delta_{T}}{1-\nu}-\frac{2 d_{T}}{1-\nu^{2}} \\
\mathrm{~B}_{122}=\mathrm{B}_{133}=\frac{\delta_{L}}{E}+\frac{2 \nu}{1-\nu} \frac{\delta_{T}+d_{T}}{E} & \mathrm{C}_{2233}=\mathrm{C}_{3322}=-\frac{\delta_{T}}{1-\nu}-\frac{2 \nu d_{T}}{1-\nu^{2}} \\
& \mathrm{C}_{2323}=-\frac{2 d_{T}}{1+\nu}
\end{array}\right)
$$

the other components being either zero or obtained by the symmetries (52). Therefore the jump conditions (43) read as

$$
\begin{gathered}
\llbracket u_{1}^{1} \rrbracket=\left(d_{L}+\frac{2 \nu}{1-\nu} \delta_{L}\right) \frac{\sigma_{11}^{0}}{E}+\left(\delta_{L}+\frac{2 \nu}{1-\nu}\left(\delta_{T}+d_{T}\right)\right) \frac{\sigma_{22}^{0}+\sigma_{33}^{0}}{E} \\
\left.\llbracket u_{2}^{1} \rrbracket=4 d_{S} \frac{\sigma_{21}^{0}}{E}\right) \llbracket u_{3}^{1} \rrbracket=4 d_{S} \frac{\sigma_{31}^{0}}{E} \\
\llbracket \sigma_{21}^{1} \rrbracket=\frac{\delta_{L}}{1-\nu} \frac{\partial \sigma_{11}^{0}}{\partial x_{2}}+\left(\frac{\delta_{T}}{1-\nu}+\frac{2 d_{T}}{1-\nu^{2}}\right) \frac{\partial \sigma_{22}^{0}}{\partial x_{2}}+\left(\frac{\delta_{T}}{1-\nu}+\frac{2 \nu d_{T}}{1-\nu^{2}}\right) \frac{\partial \sigma_{33}^{0}}{\partial x_{2}}+\frac{4 d_{T}}{1+\nu} \frac{\partial \sigma_{23}^{0}}{\partial x_{3}} \\
\llbracket \sigma_{31}^{1} \rrbracket=\frac{\delta_{L}}{1-\nu} \frac{\partial \sigma_{11}^{0}}{\partial x_{3}}+\left(\frac{\delta_{T}}{1-\nu}+\frac{2 d_{T}}{1-\nu^{2}}\right) \frac{\partial \sigma_{33}^{0}}{\partial x_{3}}+\left(\frac{\delta_{T}}{1-\nu}+\frac{2 \nu d_{T}}{1-\nu^{2}}\right) \frac{\partial \sigma_{22}^{0}}{\partial x_{3}}+\frac{4 d_{T}}{1+\nu} \frac{\partial \sigma_{23}^{0}}{\partial x_{2}}
\end{gathered}
$$

where it clearly appears that the effective behavior of the holes cannot be assimilated to a surface density of springs, see Remark 6 . Note also that we have considered the most symmetric case for a hole and hence that for less symmetric cases the effective behavior will be even more complex.

EXAMPLE 2: (A square array of transverse penny-shaped cracks) Let $\mathbf{a}=\mathbf{e}_{2}, \mathbf{b}=\mathbf{e}_{3}$, $\mathbf{Y}=\mathbb{R} \times(-1 / 2,1 / 2)^{2}, R<1 / 2$ and $\mathcal{D}=\{0\} \times \mathbf{D}_{R}$ where $\mathbf{D}_{R}$ denotes the disk $\left\{y_{2}^{2}+y_{3}^{2} \leq R^{2}\right\}$. Thus the defect is a penny-shaped crack of radius $R$ in the plane $\left(\mathbf{e}_{2}, \mathbf{e}_{3}\right)$, cf Figure 5 . The unit normal to the crack is $\mathbf{e}_{1}$ and $\operatorname{vol}(\mathcal{D})=0$. Consequently, we deduce from Proposition 4 (see also the proof) that $\mathbf{T}^{22}=\mathbf{T}^{23}=\mathbf{T}^{32}=\mathbf{T}^{33}=\mathbf{0}$. The unique non zero elementary stress fields are $\mathbf{T}^{11}, \mathbf{T}^{12}=\mathbf{T}^{21}$ and $\mathbf{T}^{13}=\mathbf{T}^{31}$. Moreover, since the material is isotropic, we can follow the procedure of Léné (1984) to check that, by symmetry, $\mathrm{D}_{1212}=\mathrm{D}_{1313}:=d_{T} / E>0$, $\mathrm{D}_{1111}:=d_{L} / E>0$ and all other coefficients which are not deduced from them by (50) vanish. In other words, the damage compliance tensor is given by

$$
\mathrm{D} \boldsymbol{\sigma} \cdot \boldsymbol{\sigma}=\frac{d_{L}}{E} \sigma_{11}^{2}+\frac{4 d_{T}}{E} \sigma_{1 \alpha} \sigma_{1 \alpha}
$$



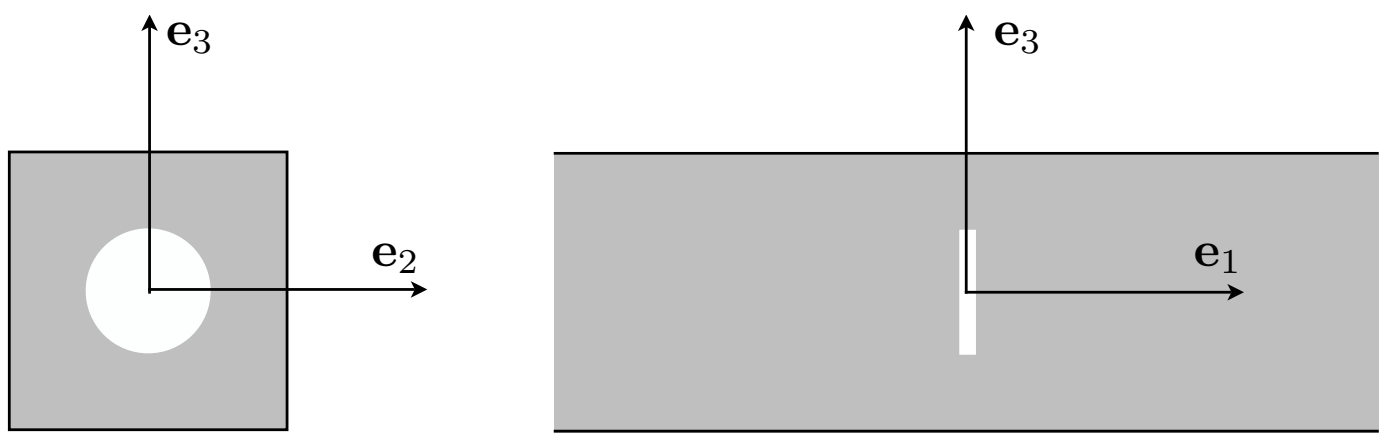

FiguRE 5. Case when the defect is a penny-shaped crack in the plane $y_{1}=0$

where the dimensionless coefficients $d_{L}$ and $d_{T}$ are increasing functions of the radius $R$ of the crack.

Since PD $=0$, by virtue of Proposition 5, the tensor $C$ vanish and $B$ is given by

$$
\mathrm{B}=\frac{d_{L}}{E} \mathbf{e}_{1} \otimes \mathbf{e}_{1} \otimes \mathbf{e}_{1}+\frac{d_{T}}{E} \mathbf{e}_{\alpha} \otimes\left(\mathbf{e}_{1} \otimes_{s} \mathbf{e}_{\alpha}\right) .
$$

Therefore, the jump conditions (43) become

$$
\llbracket \mathbf{u}^{1} \rrbracket=\frac{d_{L}}{E} \sigma_{11}^{0} \mathbf{e}_{1}+\frac{4 d_{T}}{E} \sigma_{\alpha 1}^{0} \mathbf{e}_{\alpha}, \quad \llbracket \boldsymbol{\sigma}^{1} \mathbf{e}_{1} \rrbracket=\mathbf{0},
$$

which correspond to Robin's transmission conditions, see Remark 6. In other words, this array of cracks behave like a surface density of normal and tangential springs whose compliance is equal to $\epsilon d_{L} / E$ and $4 \epsilon d_{T} / E$, respectively. It is striking to see the difference with the case of a spherical hole. We have obtained here the simplest effective behavior. That example could suggest that the effective behavior of planar cracks is always that of a surface density of springs. The next example, where we simply change the orientation of the cracks, prove the converse.

Remark 7. The jump conditions (70) have been obtained without taking account of the non interpenetration conditions of the lips of the crack. In the present case, the conditions (32) read as

$$
\hat{\tau}_{21}+\sigma_{21}^{0}=\hat{\tau}_{31}+\sigma_{31}^{0}=0, \quad \llbracket \hat{v}_{1} \rrbracket \geq 0, \quad \hat{\tau}_{11}+\sigma_{11}^{0} \leq 0, \quad\left(\hat{\tau}_{11}+\sigma_{11}^{0}\right) \llbracket \hat{v}_{1} \rrbracket=0 .
$$

It is easy to check that the solution is then

$$
\hat{\mathbf{v}}=\left\langle\sigma_{11}^{0}\right\rangle \mathbf{V}^{\mathbf{1 1}}+2 \sigma_{21}^{0} \mathbf{V}^{21}+2 \sigma_{31}^{0} \mathbf{V}^{31}, \quad \hat{\boldsymbol{\tau}}=\left\langle\sigma_{11}^{0}\right\rangle \mathbf{T}^{\mathbf{1 1}}+2 \sigma_{21}^{0} \mathbf{T}^{21}+2 \sigma_{31}^{0} \mathbf{T}^{31}
$$

where $\langle\cdot\rangle$ denotes the positive part and the $\mathbf{V}^{i j}$ 's and $\mathbf{T}^{i j}$ 's are the solutions of the elementary problems without non interpenetration conditions. Accordingly, the effective jump conditions become

$$
\llbracket \mathbf{u}^{1} \rrbracket=\frac{d_{L}}{E}\left\langle\sigma_{11}^{0}\right\rangle \mathbf{e}_{1}+\frac{4 d_{T}}{E} \sigma_{\alpha 1}^{0} \mathbf{e}_{\alpha}, \quad \llbracket \boldsymbol{\sigma}^{1} \mathbf{e}_{1} \rrbracket=\mathbf{0}
$$

and we see that the order 1 normal displacement is discontinuous only in tension. 
EXAMPLE 3: (A square array of longitudinal penny-shaped cracks) Let us change the orientation of the penny-shaped cracks and consider now that they are in the plane $\left(\mathbf{e}_{1}, \mathbf{e}_{2}\right)$, i.e. $\mathcal{D}=\mathbf{D}_{R} \times\{0\}$ with $\mathbf{D}_{R}=\left\{y_{1}^{2}+y_{2}^{2} \leq R^{2}\right\}$, cf Figure 6 . Hence the normal vector to the crack is
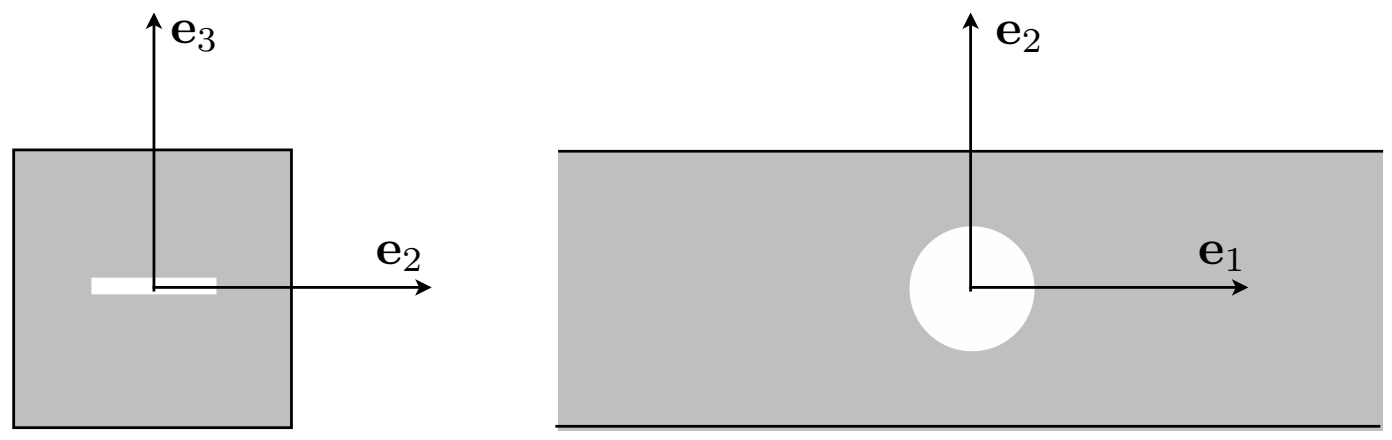

Figure 6 . Case when the defect is a penny-shaped crack in the plane $y_{3}=0$

$\mathbf{e}_{3}$ and we deduce from Proposition 4 that $\mathbf{T}^{11}=\mathbf{T}^{12}=\mathbf{T}^{21}=\mathbf{T}^{22}=\mathbf{0}$. The unique non zero elementary stress fields are $\mathbf{T}^{33}, \mathbf{T}^{13}=\mathbf{T}^{31}$ and $\mathbf{T}^{23}=\mathbf{T}^{32}$. Moreover, by symmetry, we have $\mathrm{D}_{1313}:=d_{1} / E>0, \mathrm{D}_{2323}:=d_{2} / E>0$ and $\mathrm{D}_{3333}:=d_{3} / E>0$ and all other coefficients which are not deduced from them by (50) vanish. In other words, the damage compliance tensor is given by

$$
\mathrm{D} \boldsymbol{\sigma} \cdot \boldsymbol{\sigma}=\frac{4 d_{1}}{E} \sigma_{13}^{2}+\frac{4 d_{2}}{E} \sigma_{23}^{2}+\frac{d_{3}}{E} \sigma_{33}^{2}
$$

where the three dimensionless coefficients $d_{i}$ are increasing functions of the radius $R$ of the crack. (Note that the directions 2 and 3 are no more equivalent.)

Inserting (72) into (54)-(55) and using (59) give the tensors B and C. We obtain

$$
\begin{gathered}
\mathrm{B}_{133}=\frac{\nu}{1-\nu} \frac{d_{3}}{E}, \quad \mathrm{~B}_{313}=\frac{2 d_{1}}{E} \\
\mathrm{C}_{2233}=-\frac{\nu d_{3}}{1-\nu^{2}}, \quad \mathrm{C}_{3333}=-\frac{d_{3}}{1-\nu^{2}}, \quad \mathrm{C}_{2323}=-\frac{2 d_{2}}{1+\nu}
\end{gathered}
$$

the other components being either zero or obtained by the symmetries (52). Therefore the jump conditions (43) read as

$$
\begin{gathered}
\llbracket \mathbf{u}^{1} \rrbracket=\frac{\nu d_{3}}{1-\nu} \frac{\sigma_{33}^{0}}{E} \mathbf{e}_{1}+4 d_{1} \frac{\sigma_{13}^{0}}{E} \mathbf{e}_{3} \\
\llbracket \sigma_{21}^{1} \rrbracket=\frac{\nu d_{3}}{1-\nu^{2}} \frac{\partial \sigma_{33}^{0}}{\partial x_{2}}+\frac{4 d_{2}}{1+\nu} \frac{\partial \sigma_{23}^{0}}{\partial x_{3}}, \quad \llbracket \sigma_{31}^{1} \rrbracket=\frac{4 d_{2}}{1+\nu} \frac{\partial \sigma_{23}^{0}}{\partial x_{2}}+\frac{d_{3}}{1-\nu^{2}} \frac{\partial \sigma_{33}^{0}}{\partial x_{3}} .
\end{gathered}
$$

We see that the jump conditions are now of Ventcel-type and that the previous case was an "accident". 


\section{Conclusion}

We have obtained that the order 1 transmission conditions across the interface, conditions which give the macroscopic influence of periodically distributed micro-holes or micro-cracks on the interface, are in general of Ventcel-type with discontinuity of both the displacement and the stress vector. The coefficients entering in the jump conditions are obtained by solving first six elementary elastic problems characteristic of the defect distribution. Those problems, posed on the infinite representative cell, give us in return the effective damage compliance tensor from which we deduce the desired coefficients. It appears that, even if the hole, the cell and the material have the highest degree of symmetry, the effective behavior of the interface is rather complex and far to be that of a surface density of springs. Comparatively, the simplest situation is that of planar transversal cracks. From a theoretical viewpoint, an interesting perspective is to interpret these results in terms of surface energy and to propose a variational formulation of the effective outer problem in the spirit of Abdelmoula and Marigo (2000). From a practical viewpoint, it should be interesting to quantify all these results by computing, for instance by the finite element method, the effective damage compliance tensor and the coefficients entering in the transmission conditions for several families of defects.

Acknowledgments. This work was partially supported by the French Agence Nationale de la Recherche (ANR), under grant epsilon (BLAN08-2_312370) "Domain decomposition and multiscale computations of singularities in mechanical structures"

\section{REFERENCES}

Abdelmoula, R., M. Coutris, and J.-J. Marigo: 1998, 'Comportement asymptotique d'une interphase élastique mince'. C. R. Acad. Sci. Paris Série II b 326(4), 237-242.

Abdelmoula, R. and J. J. Marigo: 2000, 'The effective behavior of a fiber bridged crack'. J. Mech. Phys. Solids 48(11), $2419-2444$.

Andrieux, S., Y. Bamberger, and J.-J. Marigo: 1986, 'Un modèle de matériau microfissuré pour les bétons et les roches'. Journal de Mécanique Théorique et Appliquée 5(3), 471-513.

Benveniste, Y.: 2006, 'A general interface model for a three-dimensional curved thin anisotropic interphase between two anisotropic media'. J. Mech. Phys. Solids 54(4), $708-734$.

Benveniste, Y. and T. Miloh: 2001, 'Imperfect soft and stiff interfaces in two-dimensional elasticity'. Mechanics of Materials 33(6), 309 - 323.

Bessoud, A. L., F. Krasucki, and G. Michaille: 2009, 'Multi-materials with strong interface: variational modelings'. Asymptotic Analysis 61(1), 1-19.

Bessoud, A.-L., F. Krasucki, and M. Serpilli: 2008, 'Plate-like and shell-like inclusions with high rigidity'. Comptes Rendus Mathematique 346(11-12), 697 - 702.

Bilteryst, F. and J.-J. Marigo: 2003, 'An energy based analysis of the pull-out problem'. Eur. J. Mech. - A/Solids 22(1), $55-69$.

Caillerie, D.: 1980, 'The effect of a thin inclusion of high rigidity in an elastic body'. Math. Methods Appl. Sci. 2, $251-270$.

Devries, F., H. Dumontet, G. Duvaut, and F. Lené: 1989, 'Homogenization and damage for composite structures'. Int. J. Numer. Methods Engrg 27, 285-298.

Dumontet, H.: 1990, Homogénéisation et effets de bords dans les matériaux composites, Thèse de doctorat d'État. Paris: Université Pierre et Marie Curie. 
Geymonat, G., F. Krasucki, and S. Lenci: 1999, 'Mathematical analysis of a bonded joint with a soft thin adhesive'. Math. Mech. Solids 4(2), 201-225.

Huy, H. P. and E. Sanchez-Palencia: 1974, 'Phénomène de transmission à travers des couches minces de conductivité élevée'. , J. Math. Anal. Appl. 47, 284-309.

Klarbring, A. and A. B. Movchan: 1998, 'Asymptotic modelling of adhesive joints'. Mechanics of Materials 28(1-4), 137 - 145.

Krasucki, F. and S. Lenci: 2000a, 'Analysis of interfaces of variable stiffness'. Int. J. Solids Struct. 37, 3619-3632. Krasucki, F. and S. Lenci: 2000b, 'Yield design of bonded joints'. Eur. J. Mech. - A/Solids 19(4), 649 - 667.

Lemrabet, K.: 1987, 'Le problème de Ventcel pour le système de l'élasticité dans un domaine de $\mathbb{R}^{3}$ '. C.R.Acad. Sci. Paris Série I 304(6), 151-154.

Léné, F.: 1984, Contribution à l'etude des matériaux composites et de leur endommagement, Thèse de doctorat d'État. Paris: Université Pierre et Marie Curie.

Licht, C. and G. Michaille: 1997, 'A modelling of elastic adhesive bonded joints'. Adv. Math. Sci. Appl. 7(2), 711-740.

Marigo, J.-J.: 1985, 'Modelling of brittle and fatigue damage for elastic material by growth of microvoids'. Eng. Fracture Mech. 21(4), 861-874.

Michel, J.-C., H. Moulinec, and P. Suquet: 1999, 'Effective properties of composite materials with periodic microstructure: a computational approach'. Comput. Methods Appl. Mech. Engrg 172, 109-143.

Nguetseng, G.: 1985, 'Problèmes d'écrans perforés pour l'équation de Laplace'. RAIRO Modél. Math. Anal. Numér. 19(1), 33-63.

Nguetseng, G. and E. Sanchez-Palencia: 1986, 'Stress concentration for defects distributed near a surface'. In: P. Ladevèze (ed.): Local Effects in the Analysis of Structures. Elsevier, Amsterdam.

Rose, L. R. F.: 1987, 'Crack reinforcement by distributed springs'. J. Mech. Phys. Solids 35(4), $383-405$.

Sanchez-Palencia, E.: 1980, Non-homogenous Media and Vibration Theory. Springer Verlag.

Sanchez-Palencia, E.: 1986, 'Elastic body with defects distributed near a surface'. In: Homogenization Techniques for Composite Media. Springer Verlag.

Suquet, P.: 1987, 'Elements of homogenization for inelastic solid mechanics'. In: E. Sanchez-Palencia and A. Zaoui (eds.): Homogenization Techniques for Composite Media, Vol. 272 of Lecture Notes in Physics. Springer Verlag, pp. 193-278.

Ventcel, A. D.: 1959, 'On boundary conditions for multi-dimensional diffusion processes'. Theor. Probability Appl. 4, 164-177.

Laboratoire de Mécanique des Solides, École Polytechnique, F91128 Palaiseau Cedex

E-mail address: marigo@lms.polytechnique.fr

Institut de Mathématiques Appliquées, Université de Toulon et du Var, F83957 La Garde Cedex

E-mail address: pideri@univ-tln.fr 